To appear in Ap.J.

\title{
Pre-Main Sequence variables in the VMR-D : identification of T Tauri-like accreting protostars through Spitzer-IRAC variability
}

\author{
T.Giannini ${ }^{1}$, D.Lorenzetti ${ }^{1}$, D.Elia ${ }^{2,3}$, F.Strafella ${ }^{3}$, M.De Luca ${ }^{1,4}$, G.Fazio ${ }^{5}$, M.Marengo ${ }^{5}$, \\ B.Nisini ${ }^{1}$, and H.A.Smith ${ }^{5}$
}

\begin{abstract}
We present a study of the infrared variability of young stellar objects by means of two Spitzer-IRAC images of the Vela Molecular Cloud D (VMR-D) obtained in observations separated in time by about six months. By using the same space-born IR instrumentation, this study eliminates all the unwanted effects due to differences in sensitivity, confusion, saturation, calibration, and filter band-passes, issues that are usually unavoidable when comparing catalogs obtained from different instruments. The VMR-D map covers about $1.5 \mathrm{deg}^{2}$ of a site where star formation is actively ongoing. We are interested in accreting pre-main sequence variables whose luminosity variations are due to intermittent events of disk accretion (i.e. active $\mathrm{T}$ Tauri stars and EXor type objects). The variable objects have been selected from a catalog of more than 170,000 sources detected at a $\mathrm{S} / \mathrm{N}$ ratio $\geq 5$. We then searched the sample of variables for ones whose photometric properties such as IR excess, color-magnitude relationships, and spectral energy distribution, are as close as possible to those of known EXor's. Indeed, these latter are monitored in a more systematic way than T Tauri stars and the mechanisms that regulate the observed phenomenology are exactly the
\end{abstract}

\footnotetext{
${ }^{1}$ INAF - Osservatorio Astronomico di Roma, via Frascati 33, I-00040 Monte Porzio, Italy, giannini, dloren,nisini,deluca@oa-roma.inaf.it

${ }^{2}$ Universidade de Lisboa, Facultade de Ciencias, Centro de Astronomia e Astrofisica da Universidade de Lisboa, Observatorio Astronomico de Lisboa, Tapada da Ajuda1349-018, Lisboa, Portugal,eliad@oal.ul.pt

${ }^{3}$ Dipartimento di Fisica, Univ.del Salento, CP 193, I-73100 Lecce, Italy, eliad,francesco.strafella@le.infn.it

${ }^{4}$ LERMA-LRA, UMR 8112, CNRS, Observatoire de Paris and Ecole Normale Suprieure, 24 Rue Lhomond, 75231 Paris, France, massimo.de.luca@lra.ens.fr

${ }^{5}$ Harvard-Smithsonian Center for Astrophysics, Cambridge, MA 02138, mmarengo@cfa.harvard.edu, hsmith@cfa.harvard.edu
} 
same. Hence the modalities of the EXor behaviour is adopted as driving criterium for selecting variables in general. We ultimately selected 19 bona fide candidates that constitute a well-defined sample of new variable targets for further investigation (monitoring, spectroscopy). Out of these, 10 sources present a Spitzer MIPS $24 \mu \mathrm{m}$ counterpart, and have been classified as 3 Class I, 5 flat spectrum and 2 Class II objects, while the spectral energy distribution of the other 9 sources is compatible with evolutionary phases older than Class I. This is consistent with what is known about the small sample of known EXor's, whose properties have driven the present selection and suggests that the accretion flaring or EXor stage might come as a ClassI/ClassII transition. We present also new prescriptions that can be useful in future searches for accretion variables in large IR databases.

Subject headings: catalogs - stars: pre-main sequence - stars: variable: other stars: formation - infrared: stars - infrared: ISM

\section{Introduction}

Young stellar objects (YSO's) from low to intermediate mass $\left(0.5-8 \mathrm{M}_{\odot}\right)$ accumulate the majority of their final mass during the so-called main accretion phase lasting about $10^{5}$ years. After this period they continue to accrete, although at lower rates. According to a commonly accepted view, material that falls onto the circumstellar disk crosses the viscous part of the disk itself and finally jumps onto the stellar surface through the magnetic interconnection lines (Shu et al. 1994). These accretion phenomena are observationally signaled by intermittent outbursts (usually detected in the optical and/or in the near-IR bands) due to the sudden increase of the mass accretion rate by orders-of-magnitude (Hartmann \& Kenyon 1985). These events are quite common during the Pre-Main Sequence life (T Tauri and Herbig Ae/Be stars) and, when some recurrent flaring events are recognizable in their light curves, they are called FUor or EXor outbursts depending on their intensity and duration (Herbig 1989; Hartmann \& Kenyon 1996). FUor's are rarer, and experience particurlarly intense outbursts (5-6 mag) followed by long periods (tens of years) during which the object remains bright. Conversely, typical EXor variability (Herbig 2007, 2008; Lorenzetti et al. 2006, 2007, 2009, hereinafter L07, L09) and the variability of accreting T Tauri stars is characterized by less intense (3-4 mag) and more short-lived outbursts (from months to a year) superposed on longer periods (few years) of quiescence. Historically, all the known accreting T Tauri stars, FUor's, and EXor's were first identified in the visual bands; as a consequence, being quite un-extincted, they have been usually associated with 
the last phases of pre-main sequence evolution. However, there is no physical reason that prevents the associated phenomena from occurring as well during more embedded, and consequently earlier, phases. In fact, an increasing number of similar objects has been identified and studied (e.g. Hodapp et al. 1996, 1999; Reipurth \& Aspin 2004; Kóspál et al. 2007; Sicilia-Aguilar et al. 2008). Obviously there are many kinds of variable objects besides accretion and eruptive variables: cataclysmic, pulsating (Mira), and eclipsing variables, for example. Hence, although accreting ones are expected to prevail in star formation regions, great care has to be taken evaluating possible contamination effects.

Sofar, the low-mass disk accretion variables are extensively studied only in nearby star forming regions (at distances less than 300 pc), but now Spitzer sensitivity has enlarged the accessible volume, thus allowing to investigate that population at larger distances, as well. Such a chance is relevant for investigating the interplay between low-, intermediate- and even high-mass stars formation processes occurring in the same region.

We recently used the InfraRed Array Camera (IRAC, Fazio et al. 2004) and the Multiband Imaging Photometer for Spitzer (MIPS, Rieke et al. 2004) on board of Spitzer Space Telescope (Werner et al. 2004) to survey $1.5 \mathrm{deg}^{2}$ of the Vela Molecular Cloud-D (Giannini et al. 2007), a star forming region in the southern sky on which we have accumulated a large variety of photometric (Massi et al. 1999, 2000, 2003, De Luca et al. 2007) and spectroscopic (Giannini et al. 2005; Lorenzetti et al. 2002) data, along with detailed maps of the gas (Elia et al. 2007) and dust (Massi et al. 2007) emission. The total integration time requested for the IRAC map was obtained by co-adding two individual maps, each integrating for half of the total time and separated by about one semester in time. This observational mode additionally provided us with two separate sets of data ideally suited for investigating the mid-infrared variability (over 6 months) of our sample of young objects. While this multiple epochs technique has been commonly used to acquire Spitzer/IRAC maps of star forming regions, to allow easy removal of transients and artifacts, these datasets have been seldom analyzed to study the variability of the sources. Given the features that characterize accreting T Tauri stars, EXor's and FUor's, the relatively short period between the two IRAC data sets does not seem suitable for a systematic search for FUor's, that usually remain stable for decades. Hence in the following discussion, when speaking of accreting variables we will refer to the class of active T Tauri stars or even to EXor's, given their similarities in the accretion modalities.

The advantages of our approach are many: $(i)$ the time elapsed between the two maps (6 months) is well suited for sampling the most common accretion events; (ii) because IRAC wavelengths are less affected by extinction than optical ones, IRAC allows us to enlarge the effective volume under investigation compared to optical observations, while preserving 
the needed sensitivity; ( $i$ ii) the IRAC bands (3.6, 4.5, 5.8 and $8.0 \mu \mathrm{m}$ ) cover the spectral range where disks emit the largest part of their energy; $(i v)$ a comparison between two sets of data obtained with the same instrumentation removes the confusion introduced by instrumental differences of sensitivity, beam confusion, saturation levels, calibration, and/or filter band-passes.

Our primary goal is to select valid candidates of variable accreting objects in VMR-D, a star forming region in which such a search has never before been done. Once completed, we expect that photometric and spectroscopic monitoring will be in order to confirm and analyze their nature. Secondly, we want+ to use our sample to provide useful distinguishing prescriptions for finding similar sources in other star forming regions, and thus to increase the number of known objects in a systematic way. In this respect we remind that our analysis can not account for the complete population of YSO's in accretion, since they spend the major part of their lifetime in quiescence or in a steady state. A thorough analysis in that sense, designed to select YSO's within the large Spitzer sample of midplane objects by reducing contamination from spurious sources, has been recently presented by Robitaille et al. (2008).

This paper follows the following structure: after having presented our observations (Sect.2) and defined the variable objects (Sect.3), we discuss our results (Sect.4) with emphasis on the source evolutionary stage and location inside the cloud. Finally, we present our conclusions (Sect.5).

\section{Observations}

In this paper we make use of a map of approximately $1.5 \mathrm{deg}^{2}$ of the Vela Molecular Cloud-D that we obtained with Spitzer-IRAC Cycle-3 GTO (PID: 30335; PI: G.Fazio) at 3.6, 4.5, 5.8 and $8.0 \mu \mathrm{m}$. The area chosen for mapping is the same we have investigated at other wavelengths (see Sect.1). Observations were carried out in two separate AORs on 2007 February 21 UT and 2007 July 4 UT (AOR Keys: 17606656 and 17606912, respectively) by adopting half-array cross scan offsets. The cross scans also enabled us to obtain much more reliable point source photometry in crowded regions.

A set of 256 frames, each $10.4 \mathrm{~s}$ integrated, was used, resulting in a total integration time of $20.8 \mathrm{~s}$ per pointing. In regions where bright sources were expected we obtained a few frames in High Dynamical Range (HDR) mode to obtain unsaturated fluxes. The IRAC data were reduced using the IRACproc package (Schuster et al. 2006), to obtain a single flux calibrated mosaic combining all the individual exposures in each epoch (separately), on a pixel grid of $0.8627 "$ pix. IRACproc is based on the Spitzer Science Center mosaic software MOPEX 
and provides enhanced outlier (cosmic rays) rejection. The IRAC point-source photometry was done using DAOPHOT package (Stetson 1987). Image mosaicing and all the details relative to both the PSF photometry extraction and the construction of a catalog containing more than 170,000 IRAC sources are presented in a dedicated paper (Strafella et al. 2009). Here we note that the spatial resolution of Spitzer in the IRAC bands is of the order of $2^{\prime \prime}$. Because of the different satellite orientations in the two epochs, the maps do not coincide precisely, as shown in Figures 1 and 2, moreover, band $1(3.6 \mu \mathrm{m})$ is acquired simultaneously with band $3(5.8 \mu \mathrm{m})$, while band $2(4.5 \mu \mathrm{m})$ simultaneously with band $4(8.0 \mu \mathrm{m})$; also these couples of maps are not exactly coincident although to a much lesser extent. As a result, in the following we will refer only to those sources detected in the common portion (between both different epochs and different bands), in order to have, for any considered source and for any considered band, two observations available for comparison.

\section{Identification of variable objects}

Since we are interested in selecting variable YSO's, the two independent sub-catalogues were searched using the following procedures. Firstly, the variable objects within a given band were defined with these selection criteria:

- detection in the considered band at a $\mathrm{S} / \mathrm{N}$ level $\geq 5$ in both epochs, separately

- ratio between the flux variation and its error, $(\mathrm{F} 1-\mathrm{F} 2) / \sigma(\mathrm{F} 1-\mathrm{F} 2) \geq 5$

- object brighter than a given threshold in both periods; namely $\leq 16$ mag (band 1$), \leq$ $15.5 \mathrm{mag}$ (band 2 ), $\leq 13.5 \mathrm{mag}$ (band 3 ), $\leq 12.5 \mathrm{mag}$ (band 4 )

The first criterium picks up objects associated with real detections, minimizing any contamination by unwanted effects such as spikes and artifacts. The second one allows us to select genuine variations well above the photometric errors. The third one is dictated by the completeness limits of our sub-catalogues (see Strafella et al., 2009, and Figure 3). Moreover, since our sample of selected variables will plausibly need near-IR spectroscopy to be definitely validated, from the start we decided only to select sources bright enough to be accessible with the current spectroscopic instrumentation.

Statistical results on the detection rate of variables are given in Table 1, where we tabulate, for each band, the number of the sources detected in the first epoch (column 1 , this number does not significantly change in the second epoch), the sources above the magnitude threshold (column 2) and the variables that satisfy all the three above criteria 
(column 3). Noticeably, the number of variables in the first two bands are more than double than those in band 3 and 4 .

Negative (positive) magnitude variations correspond to declining (rising) events between the first and the second epoch. In all the four bands, the number of declining sources is significantly larger than that of rising ones (see columns 5 and 6 of Table 1). This result suggests that the declining time is typically longer than the rising one roughly by a factor 1.3 .

In Figure 4 the distribution of variable sources (normalized to the total number of variables in that band) as a function of the magnitude variation is given. Each of the four distributions shows two peaks around a variation of $\sim$ 0.4-0.6 magnitudes, roughly symmetrical around zero. This effect is clearly a consequence of our second selection criterium that validates only those variations significantly larger than their own errors.

We note that the gap in the distribution tends to be filled at the longer wavelength bands, both because of the increase of the level of the minimum around zero, and because of the progressive decrease in height of the two peaks. This indicates that small percentage intensity variations occur preferentially at the longer wavelengths. A similar behavior has already been identified in the set of known eruptive young objects (although at different wavelengths, i.e.in the near-infrared bands): they are never observed to vary only in a single spectral band, and their percentage fluctuations usually decrease in amplitude with wavelength (L07).

The above considerations lead us to pick up among the objects that passed our criteria, those simultaneously variable in both bands 1 and 2, remaining in this way with 53 sources. These have been examined one by one looking for their location in the original $3.6 \mu \mathrm{m}$ mosaic and ruling out those sources located in the near proximity of very bright objects. In principle, PSF photometry should be less sensitive to contamination from nearby contributions, however artifacts associated to bright IRAC objects present different patterns in the two different epochs and this occurrence may mimic some spurious variability. As a result of such an inspection six further sources have been dropped out, hence our final sample is constituted by a total of 47 objects, which represent the $0.22 \%$ of the detections (above the magnitude threshold) in both bands. These 47 variable objects will be considered in the following and a catalog of these sources is given in Table 2. Here, IRAC magnitudes (one line for each epoch) are given along with complementary (and not contemporary) photometry of the 2MASS (JHK) and MIPS $(24 \mu \mathrm{m})$ counterparts (see below).

In order to verify whether or not the selected sources are intrinsically variable or perhaps appear as such because of some fluctuations of the local sky or background between the two 
epochs (for example inside the IR clusters, where the very localized diffuse emission may be relevant), we have plotted the variations (in band 1 and 2) of all the IRAC sources within $1^{\prime}$ from the selected variable. By doing so, we have temporary relaxed our second criterium (otherwise no other variable could be found in the neighborhood). We did not find any source whose flux fluctuation was comparable with that of the selected objects. This demonstrates that our sample is constituted by genuine variables, whose variation cannot be ascribed to any other phenomenum not related with the source itself.

\section{Results and Discussion}

\subsection{Location of the variable sources}

Some or all of the 47 selected sources could in principle be foreground or background objects not related to the young population of VMR-D. In Figure 1 the location of these sources, with respect to the distribution of the molecular gas, is shown. Most of them are seen to be distributed near the IR clusters, or near the peaks of the warm gas. We take this as confirmation that we are really tracing a young population of variable sources in VMR-D. In the Table 3 (2nd column) a flag is given for each object indicating whether it lies outside the CO contours $(\mathrm{O})$, inside $(\mathrm{I})$, or exactly within the peaks $(\mathrm{P})$. We use this information to build up a decision Table which will help to clarify the nature of the sources and provide a summary of all the evidences from our analysis. The aim of this approach is to investigate to what extent our sample presents characteristics similar to those of known EXor's, as derived from our previous studies (L07, L09) and here adopted as typical drivers for selecting disk accreting objects.

\subsection{Contamination}

In addition to the morphological analysis above, we have applied more quantitative tools to separate the YSO's from the remainder of the sources in the map. Main sequence stars and background galaxies can easily be recognized in the [8.0] vs.[4.5]-[8.0] diagram (e.g. Harvey et al. 2006), reported in Figure 5. Here the 47 variables are indicated with dots if observed in both of the two bands or with triangles if they have remained undetected at $8.0 \mu \mathrm{m}$, in which case we have considered the $3 \sigma$ upper limit.

In this diagram, the YSO's occupy the open triangle on the top-right side, while the photospheres are located to the left of the vertical line (at $[4.5]-[8.0]=0.5$ ) and the galaxies (whose locus has been established by the SWIRE ELAIS-N1 extragalactic survey, Rowan- 
Robinson et al. 2004) on the bottom-right side (e.g. Jørgensen et al. 2006, Porras et al. 2007). Noticeably, the majority of the sources detected in the two bands lie in the YSO's region; on the contrary, just a few $8.0 \mu \mathrm{m}$ upper limits are compatible with it. A significant fraction of sources fall just below the oblique dashed line: these could be either faint YSO's or external galaxies. In any case, since we cannot ascertain their nature in a definite way, we have labeled them as possible extragalactic (XGAL) sources in Table3, column 3. Similarly, sources with [4.5]-[8.0] $<0.5$ have been flagged as photospheres (PHT).

A harder class of objects to identify is the asymptotic giant branch (AGB) stars, that are Long Period Variables (LPVs) of Mira semi-regular, or irregular type, and which represent an important fraction of the population of variables sources in the plane of the Galaxy. As shown by Marengo et al. (2008), AGB's are sources with IR excesses easily detectable by IRAC. However, in the IRAC bandpasses they have numerous molecular absorption features whose strengths and transition frequencies depend on both their chemistry (essentially the atmospheric C/O ratio) and mass loss rates. Such features can strongly affect their IRAC colors, nominally expected to be those of reddened photospheres, and make them to appear very similar to those of YSO's. The timescale of AGB (Mira) variability is roughly comparable to that of EXor's, and as a result the time elapsed between our two epochs is not helpful in separating AGB stars from YSO's.

The spatial distribution of our 47 sources by association with the molecular cores reduces the contamination by AGB stars, which are not expected to cluster in star forming regions; nevertheless, some additional attempts deserve to be done to estimate how the AGB contamination affects our sample. To this end, we have constructed 3 two-color diagrams ([3.6]-[4.5] versus [5.8]-[8.0], [4.5]-[5.8] versus [5.8]-[8.0] and [3.6-4.5] versus [3.6]-[8.0]), marking on them the AGB loci as derived in Marengo et al. (2008). Then, we flagged as potential AGB's all the sources that simultaneously fall inside the 3 AGB's loci (or are in the close proximity to the contours) defined for each plot.

Very recently, Robitaille et al. (2008) have carefully investigated the issue of separating AGB's and YSO's, and provide specific criteria for characterizing each class with IRAC and MIPS $24 \mu \mathrm{m}$ photometry. One of these is that sources with [8.0]-[24] $<2.5$ are candidate AGB stars, while those with [8.0]-[24] > 2.5 are classified as YSO's. This means that sources with no MIPS $24 \mu \mathrm{m}$ detection cannot be separated by their criteria, but most likely are dominated by YSO's. By applying their criteria to our sample, only three sources (\#57549, 85679 and 154688) can be identified as AGB candidates. They reassuringly already belong to the sample we have flagged as potential AGB's on the basis of the IRAC color-color diagrams. In Table 3 (column 3) the candidate AGB's are labeled.

Finally, a considerable number of M-type dwarfs are expected in the foreground of 
VMR-D. The possible contamination from this class of objects will be discussed further on (Sect. 4.4).

The ultimate test capable of proving the nature of the selected sources is obviously spectroscopic. Indeed, by taking a low resolution $(\mathcal{R} \sim 200-300)$ near-IR spectrum (1.0$2.5 \mu \mathrm{m}$ ) of any individual source, we would be able to determine if it is an emission line object (as all the T Tauri stars are, and all the EXor's appear to be - L09), a protostar with an increasing continuum with wavelengths, or an AGB star characterized by deep absorption bands due to water vapor at 1.4 and $1.9 \mu \mathrm{m}$ (and sometimes even at $2.5 \mu \mathrm{m}$ ) superposed on an increasing continuum. As an example, we show in Figure 6 the spectra of two bright sources belonging to a sample of protostellar candidates selected from IRAC/MIPS data in the Serpens star forming region (Winston et al. 2007). Both these sources \#35 and 71, here called win35 and win71 for simplicity) were observed during a spectral survey we are carrying out at our $1 \mathrm{~m}$ IR telescope (at Campo Imperatore - Italy). As is apparent, win35 behaves as a typical protostellar source, while win71 presents unequivocally (and confirmed in two repeated spectra) the deep $\mathrm{H}_{2} \mathrm{O}$ absorption bands more typical of an AGB star (the possibility that this source is a protostar of late spectral type can be disregarded because of its brightness). The same conclusions have indeed reached by Winston et al. (2009), who have recently undertaken a near-infrared spectroscopic survey of the YSO's in Serpens. These examples serve to illustrate $(i)$ how the IR colors alone, without any further evaluation, can lead to possible misidentifications, and ( $i i)$ that near-IR spectroscopy is an effective means of refining the identifications based on IRAC/MIPS colors alone.

\subsection{IRAC colors}

The EXor's monitored so far in the near-IR $(1-2.5 \mu \mathrm{m})$ present two main photometric characteristics (L07): (i) as most intrinsic variables, they become bluer during the outburst and redder while they fade; (ii) the amplitude of the variations decrease with increasing the wavelength. These conclusions are based on observations of well-defined variability events, namely, those where the full amplitude of the variation is $\Delta \mathrm{mag} \gtrsim 1 \mathrm{mag}$, and not to marginal fluctuations usually associated with randomly sampled events. During the outburst phase a peak at a relatively high temperature becomes more and more evident (blueing), while marginal fluctuations can be ascribed to colder contributions typical of a disk temperature stratification. In the present sample, $\Delta$ mag $\gtrsim 1$ variations are quite rare (see Figure 4). Nevertheless, with the above-mentioned caveat in mind, it is useful to search our sample for some similarities (or differences) with known EXor's, since here the lack of a systematic monitoring is compensated by approaching a large and unbiased sample in a statistical 
sense. To that end, we present in Figures $7 \mathrm{a}$ and $7 \mathrm{~b}$ the magnitude difference [mag(epoch1)mag(epoch2)] as a function of the wavelength, with variations measured between the first and the second epochs, while Figures $8 \mathrm{a}$ and $8 \mathrm{~b}$ depict the [3.6]-[4.5] vs. [4.5] color-magnitude plots of the selected sources.

From the data shown in these Figures some conclusions can be derived:

(i) from Figures $7 \mathrm{a}$ and $7 \mathrm{~b}$ we see that in 21 cases (representing $\sim 45 \%$ of the total sample) the objects amplitude decreases with increasing wavelength, as is typical of the monitored EXor's; 17 cases $(\sim 35 \%)$ show comparable variability at all wavelengths; while for the remaining 9 sources (less than 20\%) the variation appears to increase with wavelength. Noticeably, just one of these latter objects is classified as YSO by means of the color-color and color-magnitude diagrams. These results are summarized in column 4 of Table 3 with the following codes: D(ecreasing), C(onstant), I(ncreasing). They confirm that, for the majority of sources (38 out of 47), IRAC variability is consistent with that displayed by EXor's;

(ii) irrespective of the source brightness (from 15 to $10 \mathrm{mag}$ at $4.5 \mu \mathrm{m}$, see Figures $8 \mathrm{a}$ and 8b) the amplitude of the [3.6]-[4.5] color variation is substantially similar $(\lesssim 0.2 \mathrm{mag})$ for sources whose color lies in the range 0.0-1.0 mag (with only 7 exceptions of redder color);

(iii) as mentioned above, intrinsic variables in the near-IR tend to show redder and redder colors while becoming fainter, although this conclusion can only be univocally applied to sources with large fluctuations. The opposite (i.e. the colors become bluer), happens when the source becomes brighter. With reference to Figures $8 \mathrm{a}$ and $8 \mathrm{~b}$, both these variation modalities translate into a negative slope of the line connecting the two data points. This information is also listed in column 5 of Table 3, coded as -, 0, + to indicate a negative, null or positive slope of this line. The majority of sources (37 out of 47) are fully compatible with known EXor behavior, i.e are coded as - or 0.

\subsection{Spectral Energy Distributions}

We obtained the Spectral Energy Distributions (SED's) for the 47 selected variables by identifying counterparts at both shorter and longer wavelengths when possible. To identify possible counterparts in the JHK bands, we used the Two Micron All Sky Survey (2MASS) catalog (Cutri et al. 2003), looking for matches within a radius of $2^{\prime \prime}$. We identified 21 sources with a valid 2MASS detection, and the corresponding JHK magnitudes are given in Table 2 , We also searched the literature (Massi et al. 2000) for JHK images at a better sensitivity (magnitude limit is $\mathrm{K} \sim 18$ ) than 2MASS, but, unfortunately, none of them overlaps the regions with the selected variables. In the view of a future spectroscopic followup, we have 
also searched for optical counterparts: the positive detections are listed in a footnote of Table 2. To search for longer wavelength counterparts, we used the IRAS Point Source Catalog, the Midcourse Space Experiment (MSX) catalog (Price et al. 2001) and our MIPS catalog (Giannini et al. 2007). No association was found to any of our 47 sources in the former two, to within a radius of $20^{\prime \prime}$, while $24 \mu \mathrm{m}$ MIPS counterparts were found in only 13 cases using a search radius of $6^{\prime \prime}$ (also listed in Table 2). Only two of our sources (namely \#121029 and 124521) have a $70 \mu \mathrm{m}$ counterpart in a search radius of $20^{\prime \prime}$; however, the multiplicity of the IRAC sources in the MIPS $70 \mu \mathrm{m}$ beam implies that these associations are not unique, and we do not consider them in the following analysis. Statistically, 50\% of the IRAC selected variables possess a near-IR counterpart, $30 \%$ have a $24 \mu \mathrm{m}$ one, and about $20 \%$ have both.

Figures 9a and 9b show the SED's of the 47 variable sources, from 2MASS wavelengths to MIPS $24 \mu \mathrm{m}$ (when available). The IRAC fluxes from both epochs are shown in different colors; black dots represent the complementary photometry from different periods. Open squares indicate IRAC and MIPS $3 \sigma$ upper limits. For comparison, a median stellar photosphere in the spectral range K5-M5 is also plotted in each panel (Hernández et al. 2007), normalized to the flux corresponding to the shortest infrared wavelength available, namely J or $3.6 \mu \mathrm{m}$ (this latter for the sources without a 2MASS counterpart). The last two panels show the SED's of UZ Tau E and V1647 Ori: the first one (Hartmann et al. 2005) is a well known and quite unextincted nearby EXor, while the second is a more embedded (and maybe more massive) candidate observed with Spitzer/2MASS during an outbursting phase (Muzerolle et al. 2005). These distributions, that appear to be fully consistent with that expected for accretion disks (e.g. D'Alessio et al. 1999), can be used as a working template for comparing the SED's of our selected variables, and for identifying among them the most likely acccreting YSO's candidates. By examining the SED's of our variables, we can identify some objects as a late type photosphere; others show an excess (more or less pronounced) at increasing wavelengths, that is typical of a temperature stratification due to the presence of an evolved circumstellar disk or envelope. We define, as a quantitative indicator, the ratio $\mathcal{E}$ between the observed SED and the underlying median K5-M5 photosphere, both integrated from J to $8.0 \mu \mathrm{m}$. For UZ Tau E and V1647 Ori we obtain $\mathcal{E}=2.3$ and 26.8, respectively. Therefore, we conservatively suggest that accreting YSO candidates are those objects with $\mathcal{E}>1$. The result of such a criterium is seen in Table 3 (column 6), and can be summarized by saying that more than $50 \%$ of the 47 selected variables present a SED compatible with an accretion disk. We notice that none of the objects with $\mathcal{E}>1$ simultaneoulsy presents IRAC fluxes compatible with a photosphere and a MIPS excess: this implies that possible binary systems composed by a variable photospheric object and a colder companion do not contaminate our sample. 
The shapes of the SED's also suggest that a non-negligible fraction of the luminosity is emitted at wavelengths longer than those probed with our observations. Consequently, we cannot give a reliable estimate of the bolometric luminosity. The absolute values of the luminosities in the IRAC range $\left(\mathrm{L}_{I R A C}\right.$, last column of Table 3) are, on average, remarkably low (from thousandths to tenths of one solar luminosity) when compared with the IRAC estimated luminosities of known eruptive variables $\left(7.9\right.$ and $0.4 \mathrm{~L}_{\odot}$ for V1647 Ori and UZ Tau, respectively). Given the high sensitivity of IRAC, this circumstance is not surprising, since, having observed VMR-D just twice without performing a systematic monitoring, we sample primarily the numerous, low luminosity end of the stellar distribution in the cloud. The luminosities obtained in both epochs differ from each other by between 15 and $50 \%$ (with a few larger exceptions). Such variations are consistent with $\dot{M}$ fluctuations within a factor 2-4, exactly the values expected over these time-scales (L09).

As anticipated in Sect.4.2, several M-type red-dwarf stars displaying emission lines $(\mathrm{dMe})$ are expected in the foreground of VMR-D, given its distance at about $700 \mathrm{pc}$. These dMe stars present both fluxes comparable to those of our variables and flaring activity due to enhanced coronal emission. However, their SED's are fitted by a purely photospheric spectrum up to $24 \mu \mathrm{m}$ and no evidence for IR excess has been found (Riaz et al. 2006). Since a definite presence of IR excess is required by our selection, our final sample of variables is not affected by dMe contamination. We are however able to detect them. To roughly estimate a lower limit of (K-M) flaring photospheres that lie on our investigated area, we notice that 10 objects (i.e. about $20 \%$ of the total) have been disregarded as accreting candidates, but display SED's typical to a photospheric spectrum (see Figure 9 and Table 3 for their $\mathcal{E}$ value).

\subsection{Analogies with known eruptive variables}

Having now accumulated in Table 3 a range of independent markers for EXor behavior, or more simply of active T Tauri stars, we can now scrutinize these indicators in a systematic way in an attempt to identify the variables that behave as the young accretors do: namely, those that simultaneously present all the characteristics summarized in the last line of Table 3 and discussed in the previous Sections. After analyzing in this way each of the sources in Table 3, we conclude that 19 (boldfaced) sources out of 47 manifest the same five flags we argue are typical of EXors: $\mathrm{P}(\mathrm{I}), \mathrm{D}(\mathrm{C})$, YSO, $-(0)$, and $>1.0$. Hence, we identify these 19 as the accreting protostars candidates emerging from the present study. In particular, 6 of them have the same flags strictly ( $\mathrm{P}, \mathrm{D}, \mathrm{YSO},-,>1.0)$. Out of the remaining 29 sources, another 10 show 4 flags in accordance with the template, while 19 sources have three or fewer identifying flags. 
From an evolutionary point of view, protostars can be classified according to the value of the spectral index computed from 2 to $10 \mu \mathrm{m}$ (Greene et al. 1994); however the classification scheme does not change substantially if computed up to fluxes of $20-25 \mu \mathrm{m}$ (e.g. Rebull et al. 2007). Among the final 19 objects, 10 are detected at $24 \mu \mathrm{m}$. Of these, 3 are Class I (\# 17825, 44510, 50748), 5 are flat spectrum (\# 67878, 84520, 107546, 110128, 125801) and 2 are Class II sources (\# 131555, 133791). This indicates that the accreting flaring stage may occur earlier than the Class II phase, at least in the framework of an evolutionary scenario. For comparison, L07 have classified 2 EXor's as Class I sources (NY Ori and PV Cep), 3 as flat spectrum sources (XZ Tau, V1143 Ori and EX Lup) and 4 as Class II (UZ Tau, VY Tau, DR Tau and V1118 Ori). For the remaining 9 sources, we computed the slope of the SED up to the longest wavelength available (typically $8 \mu \mathrm{m}$ ) and consistent with the $24 \mu \mathrm{m}$ upper limit. In this way we find that 3 and 6 sources are compatible with (or older than) flat and Class II sources, respectively.

Our demography of the VMR-D cloud (Strafella et al. 2009) provides a total of 487 YSO's made up of Class I (62), flat spectrum (92) and Class II (333) objects. It is remarkable that in addition there are 171 Class III sources associated to the VMR-D. Because variables that were in quiescence during the epochs of our monitoring are missed by the present selection, we cannot reasonably attempt to estimate the duration of the intense disk accretion phase with respect to the entire PMS lifetime. We can, however, conclude that: $i$ ) by adopting our empirical approach, about $50 \%$ of the selected variables are potential active $\mathrm{T}$ Tauri stars or EXor candidates deserving of further study (mainly IR monitoring and spectroscopy); $i$ ) a value of $4 \%$ is a lower limit on the percentage of accreting variables with respect to the total protostars (from Class I to Class II sources) in VMR-D; iii) an important fraction of these 19 candidates is constituted by objects with Class I and flat spectra, a trend opposite to the young stellar population in VMR-D which, on the contrary, is dominated by Class II and III sources. This circumstance might stem from our requirement that our candidates are requested to be associated with $\mathrm{CO}$ peaks or filaments: this results in finding more embedded and younger objects. Moreover, the need for a detectable excess ( $\mathcal{E}$ flag) goes in the same direction.

\section{Concluding Remarks}

We present a catalog of objects identified as probable PMS variables, obtained by comparing two IRAC images of the star forming region VMR-D separated by six months. By analyzing the results, we reach the following conclusions:

1. The same selection criteria were applied to the data-set of both epochs aiming to find 
real detections (by removing spikes and artifacts) with genuine variations (well above the photometric errors). Recorded flux fluctuations span from $10 \%$ to more than $100 \%$.

2. Variability in the first two IRAC bands $(3.6$ and $4.5 \mu \mathrm{m})$ identifies more than twice as many objects than does variability in bands 3 and $4(5.8$ and $8.0 \mu \mathrm{m})$.

3. 47 variable objects have been identified (out of a total of 170,000 sources). We estimate the possible contamination of the sample by considering extraneous galactic or extragalactic sources by using the well-defined properties of active stars (or EXor type) with recurrent disk accretion phenomena as templates.

4. Spectral Energy Distributions were constructed from near- to mid-IR for all the 47 sources. IRAC luminosities are remarkably low (from thousandths to tenths of one solar luminosity), but this is not surprising since, having observed the region just twice without performing a systematic monitoring, we likely sample the low luminosity end of the stellar distribution.

5. 19 sources from our full sample of 47 have all the same properties that characterize known EXor objects. They are potential accretion flaring or EXor candidates and deserve further studies (mainly IR monitoring and spectroscopy) to better characterize their nature. In this latter respect we note that the brightness of the selected variables is well compatible with the sensitivity of the current infrared spectroscopic instrumentation.

6. A significant number (i.e. 8 sources) of the final 19 candidates are recognizable as Class I and flat spectrum sources (with 2 Class II objects). This suggests that the accretion flaring or EXor stage might come as a Class I/Class II transition.

7. New prescriptions are derived from our analysis that can facilitate identifying accretion variables in large IR database.

The authors would like to thank Arkady A.Arkharov and Valeri M.Larionov for providing them with the near IR spectra of the sources win35 and win70, taken at Campo Imperatore (Italy). This work is based on observations made with the Spitzer Space Telescope, which is operated by the Jet Propulsion Laboratory, California Institute of Technology under NASA contract 1407. Support for the IRAC instrument was provided by NASA under contract number 1256790 issued by JPL. 


\section{REFERENCES}

Cutri, R.M., Strutskie, M.F., Van Dyk, S. et al. 2003 Explanatory Supplement to the 2MASS All Sky Data Release (Pasadena: Caltech)

De Luca, M., Giannini, T., Lorenzetti, D. et al. 2007 A\&A 474, 863

D’Alessio, P., Calvet, N., Hartmann, L., Lizano, S. \& Cantó, J. 1999 ApJ 527, 893

Elia, D., Massi, F., Strafella, F. et al. 2007 ApJ 655, 316

Fazio, G.G., Hora, J.L., Allen, L.E. et al. 2004 ApJS 154, 10

Jørgensen, J.K., Harvey, P.M., Evans II, N.J., et al. 2006 ApJ 645, 1246

Giannini, T., Lorenzetti, D., De Luca, M. et al. 2007 ApJ 671, 470

Giannini, T., Massi, F., Podio L. et al. 2005 A\&A 433, 941

Greene, T.P., Wilking, B.A., Andrè, P., Young, E.T., Lada, C.J. 1994 ApJ 434,614

Hartmann, L. \& Kenyon, S. 1985 ApJ 299, 462

Hartmann, L. \& Kenyon, S. 1996 ARAA 34, 207

Hartmann, L., Megeath, S.T., Allen, L. et al. 2005 ApJ 629, 881

Harvey, P.M., Chapman, N., Shih-Ping, L. et al. 2006 ApJ 644, 307

Herbig, G.H. 1989 Proc. of the ESO Workshop on Low Mass Star Formation and Pre-Main Sequence Objects, ed. B. Reipurth, p.233

Herbig, G.H. 2007 AJ 133, 2679

Herbig, G.H. 2008 AJ 135, 637

Hernández, J., Hartmann, L., Megeath, T. et al. 2007 ApJ 662, 1067

Hodapp, K.W. 1999 AJ 118, 1338

Hodapp, K.W., Hora, J.L., Rayner, J.T. et al. 1996 ApJ 468, 861

Kóspál, Á., Ábrahám, P. , Acosta-Pulido, J. et al. 2007 A\&A 470, 211

Lorenzetti, D., Giannini, T., Calzoletti, L. et al. 2006 A\&A 453, 579

Lorenzetti, D., Giannini, T., Larionov, V.M. et al. 2007 ApJ 665, 1182 (L07) 
Lorenzetti, D., Giannini, T., Vitali, F. et al. 2002 ApJ 564, 839

Lorenzetti, D., Larionov, V.M., Giannini, T. et al. 2009, ApJ 693, 1056 (L09)

Marengo, M., Reiter, M. \& Fazio, G.G. 2008, in AIP Conf. Proc. 1001, IXth Torino Workshop on Evolution and Nucleosynthesis in AGB stars and the 2nd Perugia Workshop on Nuclear Astrophysics. (New York, AIP), 331

Massi, F., De Luca, M., Elia, D. et al. 2007 A\&A 466, 1013

Massi, F., Lorenzetti, D. \& Giannini, T. 2003 A\&A 399, 147

Massi, F., Lorenzetti, D., Giannini, T. \& Vitali, F. 2000 A\&A 353, 598

Massi, F., Giannini, T., Lorenzetti, D. et al. 1999 A\&AS 136, 471

Muzerolle, J., Megeath, S.T., Flaherty, K.M. et al. 2005 ApJ 620, 107

Porras, A., Jørgensen, J.K., Allen, L.E., et al. 2007 ApJ 656, 493

Price, S.D., Egan, M.P., Carey, S.J., Mizuno, D.R., \& Kuchar, T.A. 2001 AJ 121, 2819

Rebull, L.M., Stapelfeldt, K.R., Evans II, N.J et al. 2007 ApJ, 171, 447

Reipurth, B. \& Aspin, C. 2004 ApJ 606, L119

Riaz, B, Muller, D.J., \& Grizis, J.E. 2006 ApJ, 650, 1133

Rieke, G.H., Young, E.T., Engelbracht, C.W. et al. 2004 ApJS 154, 24

Robitaille, T.P., Meade, M.R., Babler, B.L. et al. 2008 AJ 136, 2413

Rowan-Robinson, M., Lari, C., Perez-Fournon, I., et al. 2004 MNRAS 351, 1290

Sicilia-Aguilar, A., Merín, B., T., Hormuth, F. \& Ábrahám, P. 2008 ApJ 673, 382

Schuster, M. T., Marengo, M., Patten, B. M. 2006 SPIE meeting, Orlando, FL, \#6720-65

Strafella, F., et al. 2009 ApJ, in preparation

Shu, F.H., Najita, J.R., Ostriker, E. et al. 1994 ApJ 429, 781

Stetson, P.B. 1987 PASP 99, 191

Werner, M.W., Roelling, T.L., Low, F.J. et al. 2004 ApJS 154, 1

Winston, E., Megeath, S.T., Wolk, S.J. et al. 2007 ApJ 669, 493 
Winston, E., Megeath, S.T., Wolk, S.J. et al. 2009 ApJ, in press (arXiv0904.1244W)

This preprint was prepared with the AAS LATEX macros v5.2. 


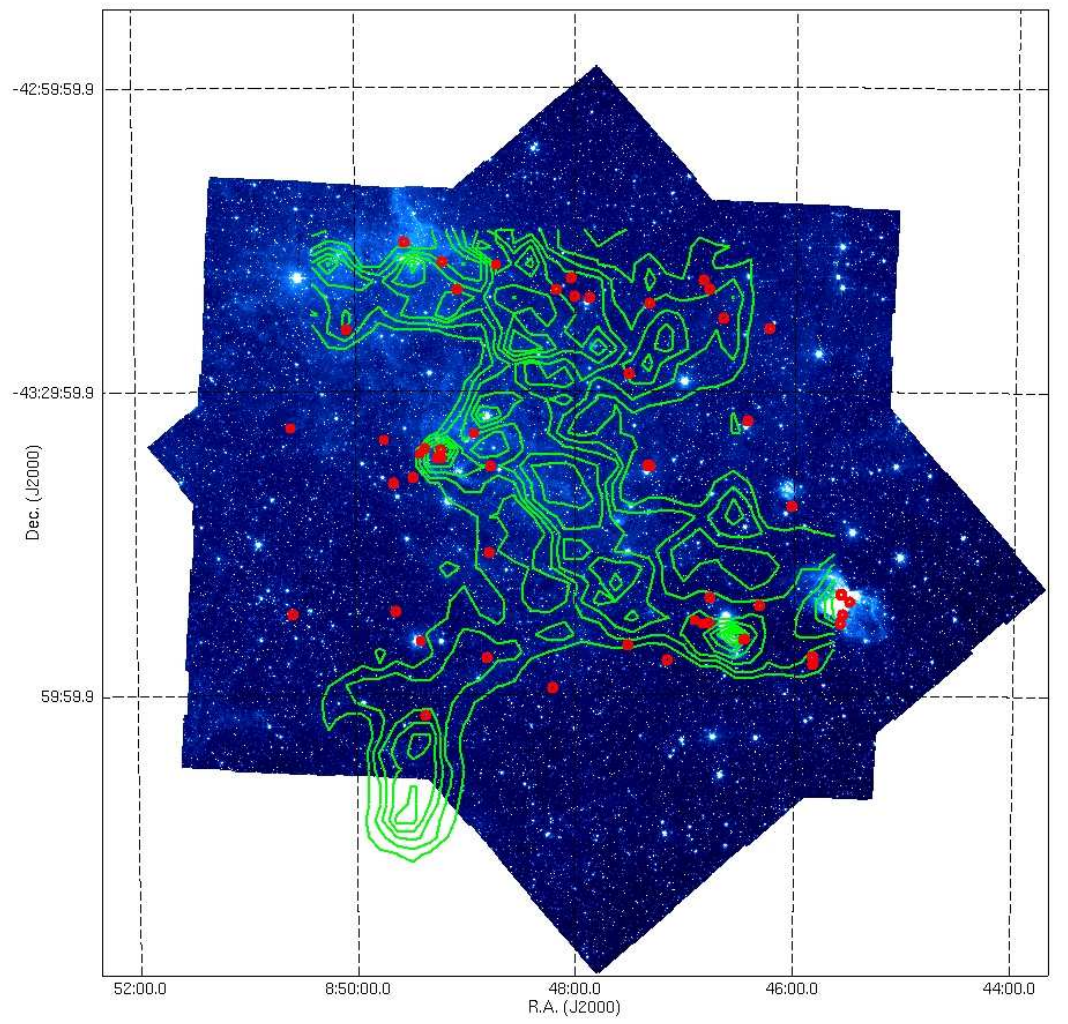

Fig. 1. - IRAC maps at $3.6 \mu \mathrm{m}$ obtained in two different epochs. Due to the satellite orientation, they are rotated in a different way. The common part is clearly recognizable. The contours of our CO map (Elia et al. 2007) are superposed (in green). The $5.8 \mu \mathrm{m}$ channel is acquired simultaneously, therefore it presents the same superposition. The location of the selected variables is shown with red dots. 


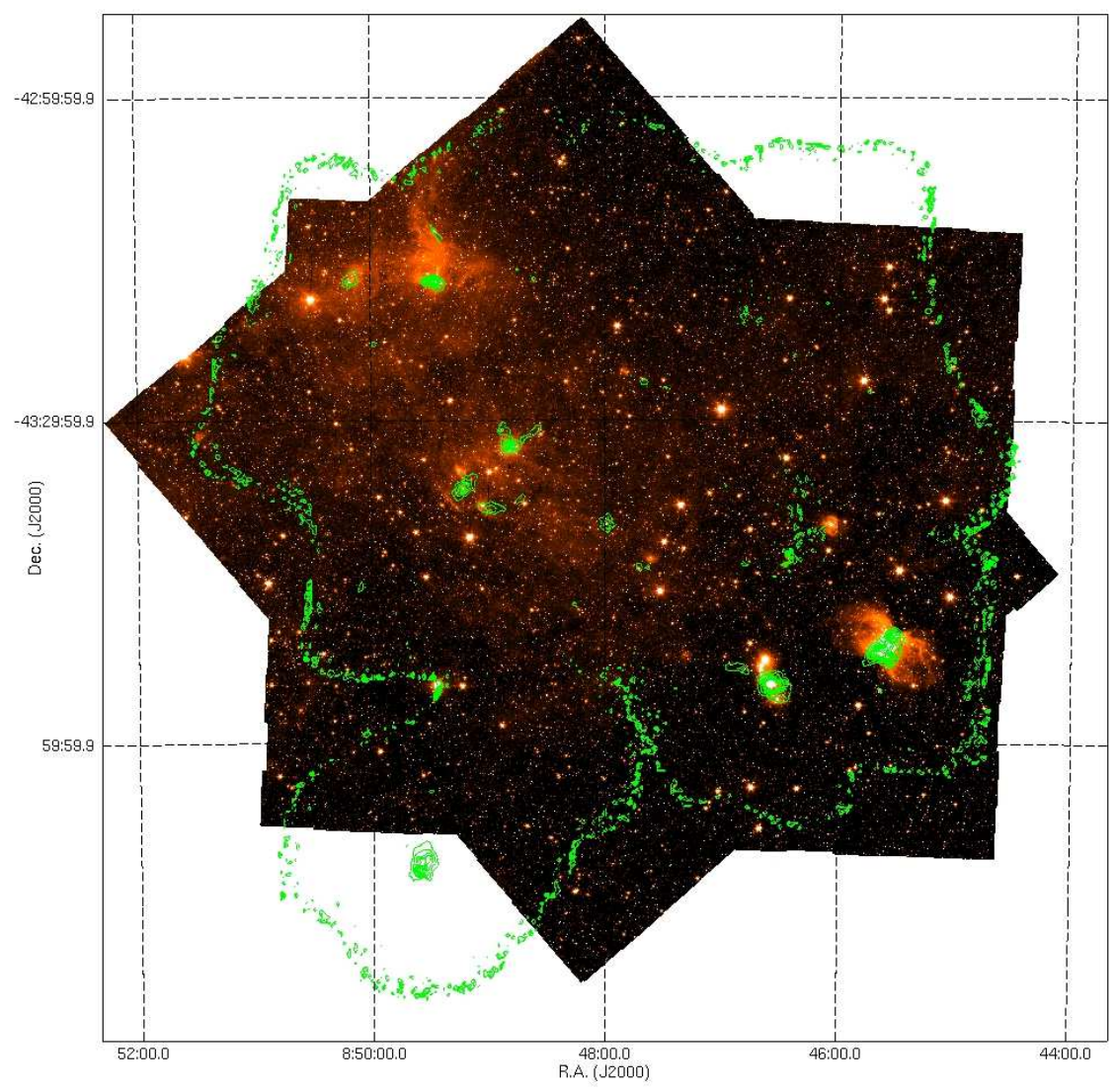

Fig. 2.- As in Figure 1, but for the $4.5 \mu \mathrm{m}$ channel (acquired simultaneously to the $8.0 \mu \mathrm{m}$ one). Notice that the common part between the two epochs is slightly different from that of channels 3.6 and $5.8 \mu \mathrm{m}$. Here, for completeness, our dust continuum map at $1.2 \mathrm{~mm}$ (Massi et al. 2007) is superposed (in green). 

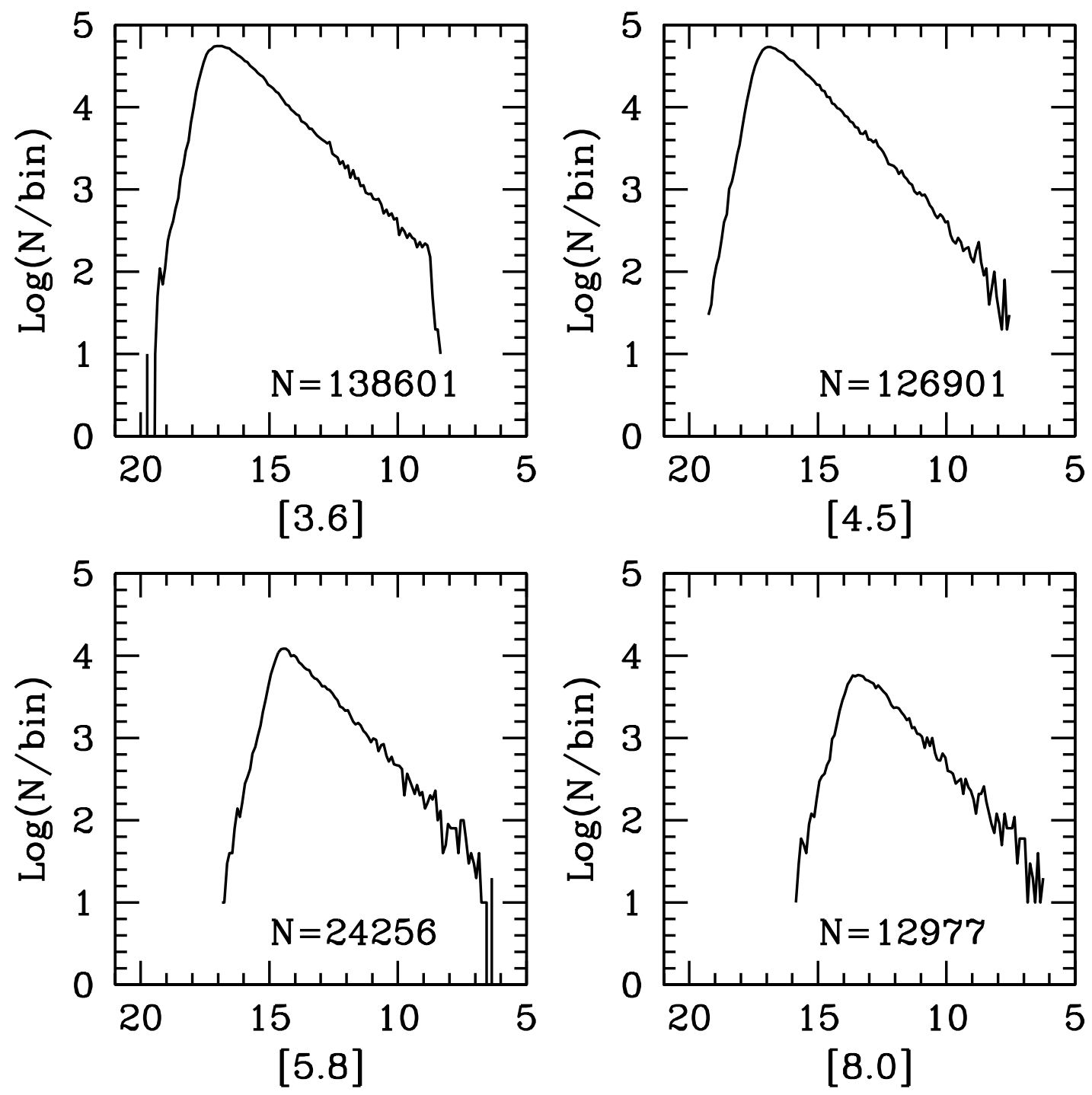

Fig. 3.- Magnitude distribution of all the IRAC sources in VMR-D as detected in the first epoch (see text). The same distribution relative to the second epoch does not present any significant difference. 


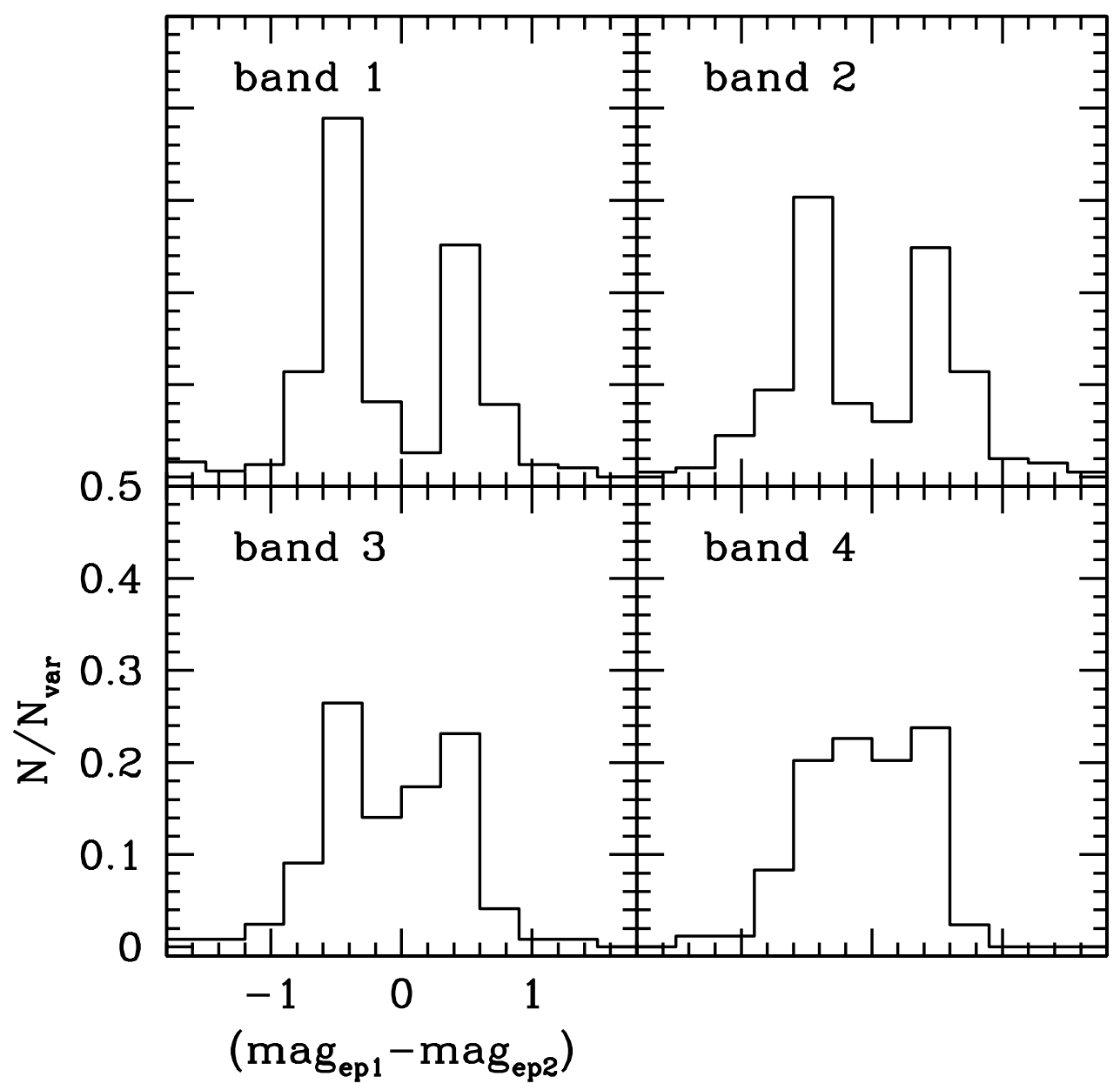

Fig. 4.- Distribution of the variable IRAC sources as a function of the magnitude variation. 


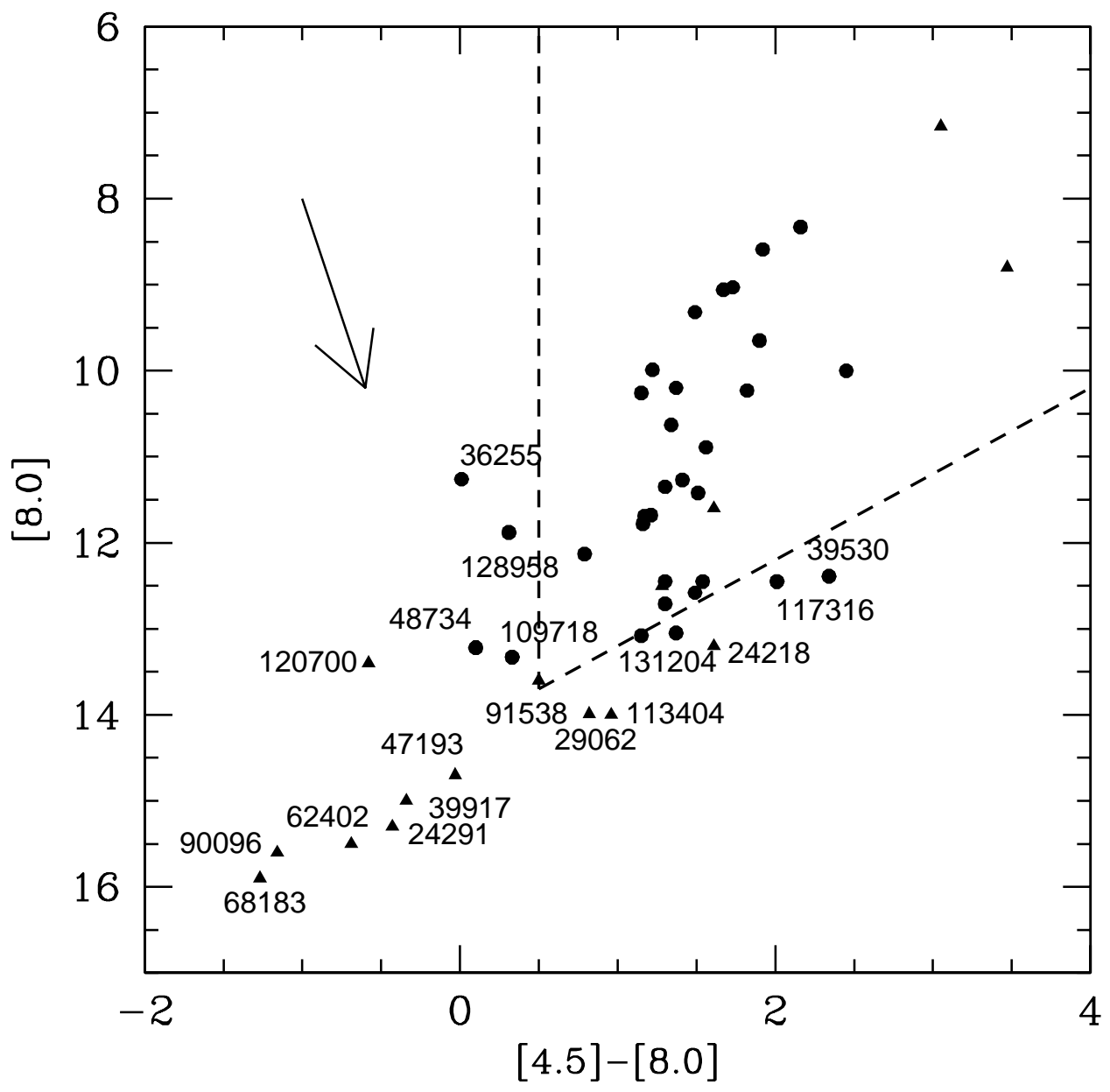

Fig. 5.- Color-magnitude diagram of the 47 selected variables. In this diagram YSO's are located between the lines $[4.5]-[8.0]>0.5$ and $[8.0]<14-([4.5]-[8.0])$, i.e. the open triangle at the top-right. Dots represent objects detected in both considered bands; triangles indicate the upper limits at $8.0 \mu \mathrm{m}$ : therefore they should imagined to point toward the bottom-left corner of the figure. We have labeled the sources which lie outside the region occupied by YSO's, also flagged as extragalactic (XGAL) or photospheres (PHT) in Table 3. The arrow shows the effect of the extinction for $\mathrm{A}_{V}=50 \mathrm{mag}$. 
Win 35

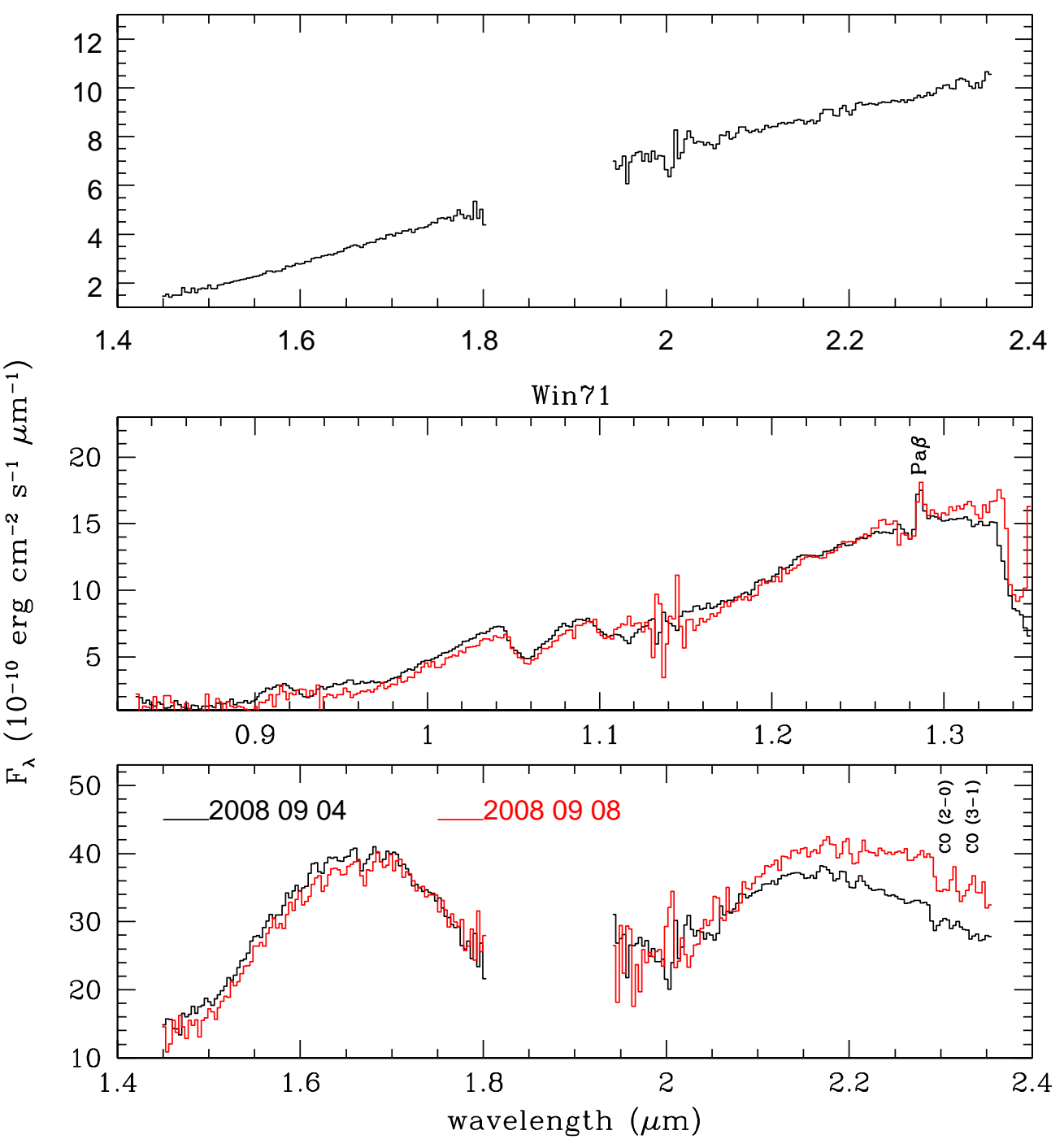

Fig. 6.- Near IR spectra of the sources win 35 (top) and win 71 (bottom). 


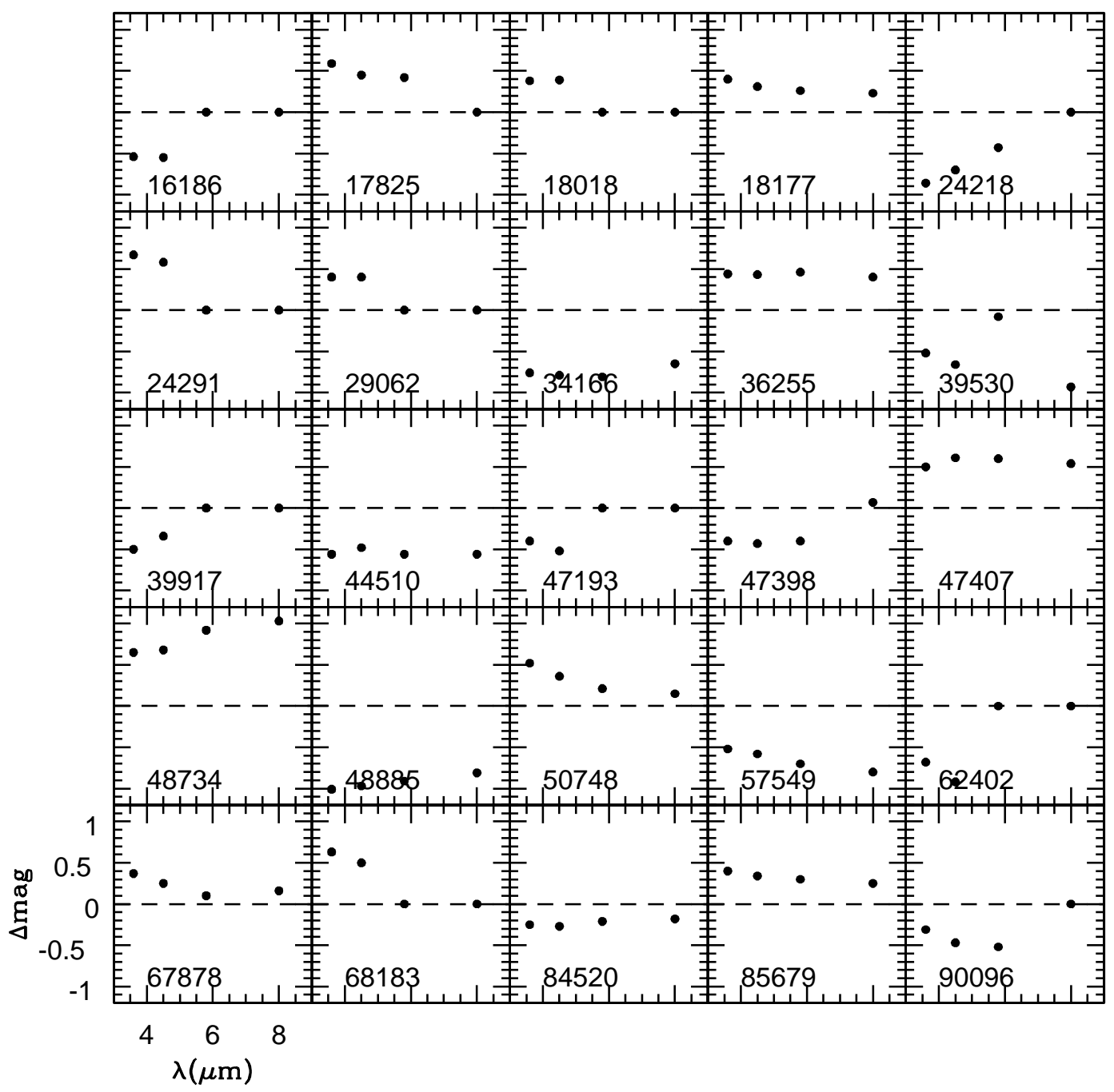

Fig. 7.- a). Magnitude variation of the 47 sources in the two epochs, $\Delta \operatorname{mag}=[\operatorname{mag}($ epoch1)mag(epoch2)], as a function of the observed wavelength. 


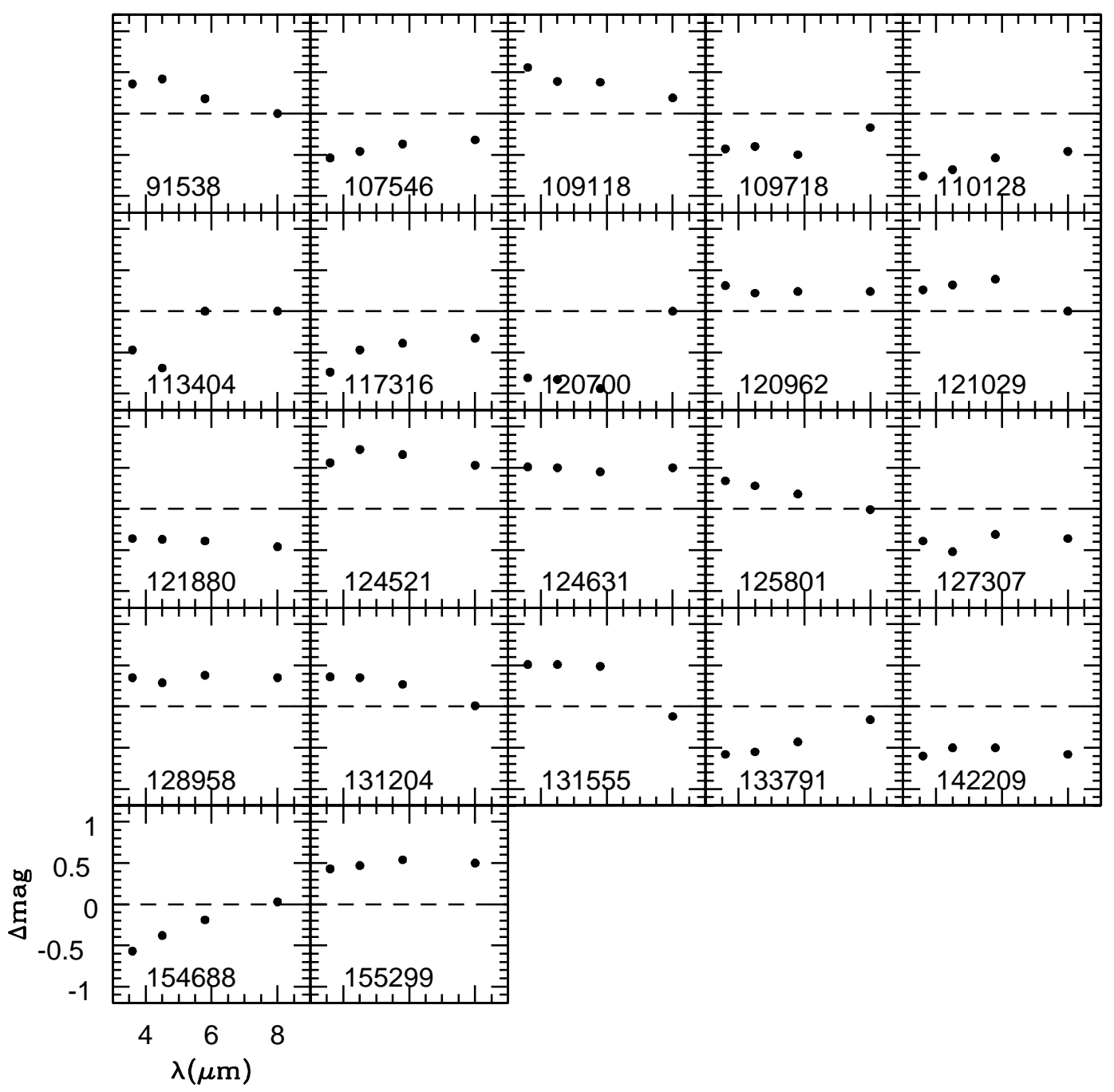

Fig. 7.- b). As Figure 7b, continued. 


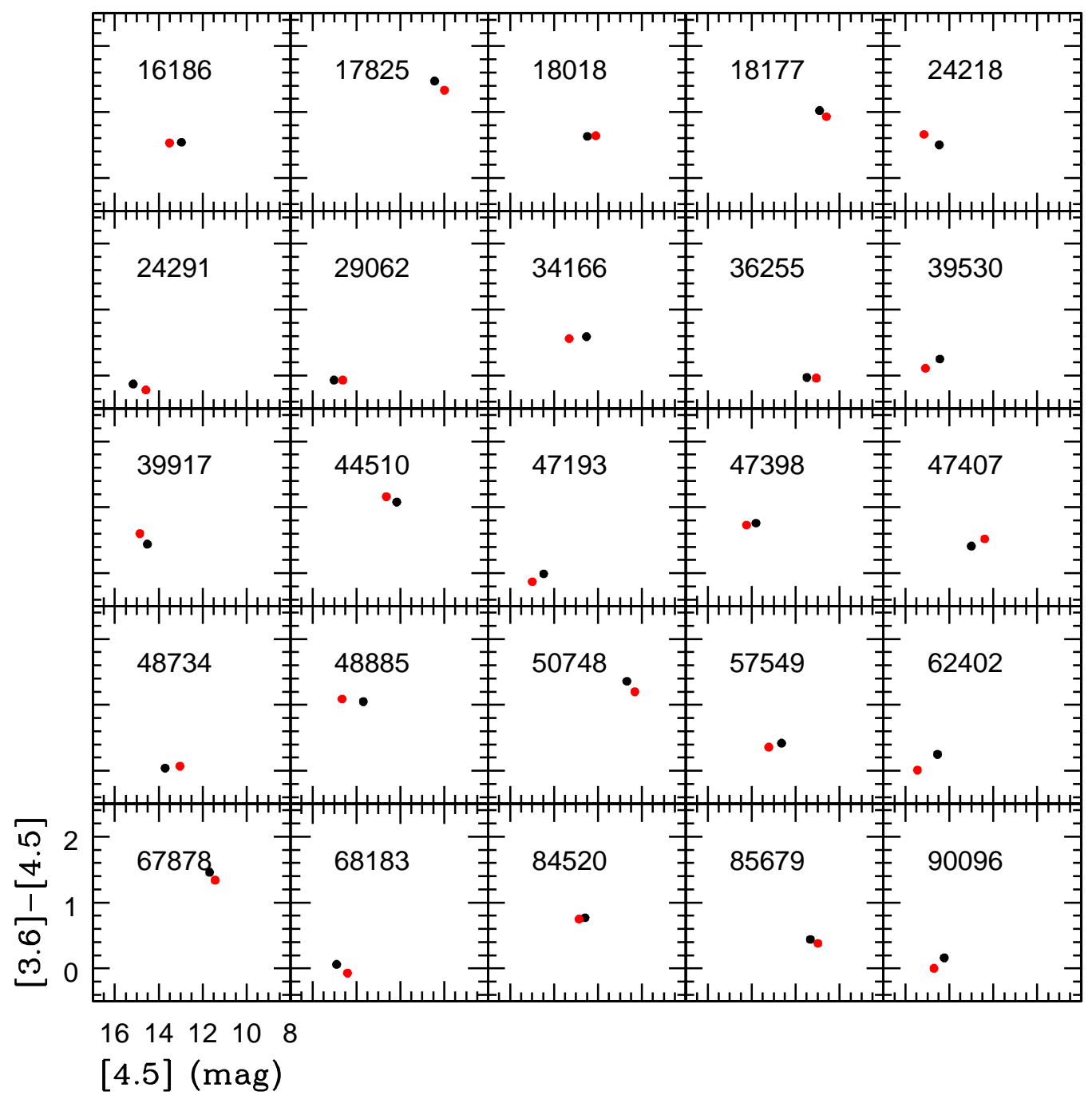

Fig. 8.- a). Observed color [3.6]-[4.5] vs. [4.5] magnitude of the 47 selected sources in both epochs. Black (red) dot refers to the first (second) epoch. 


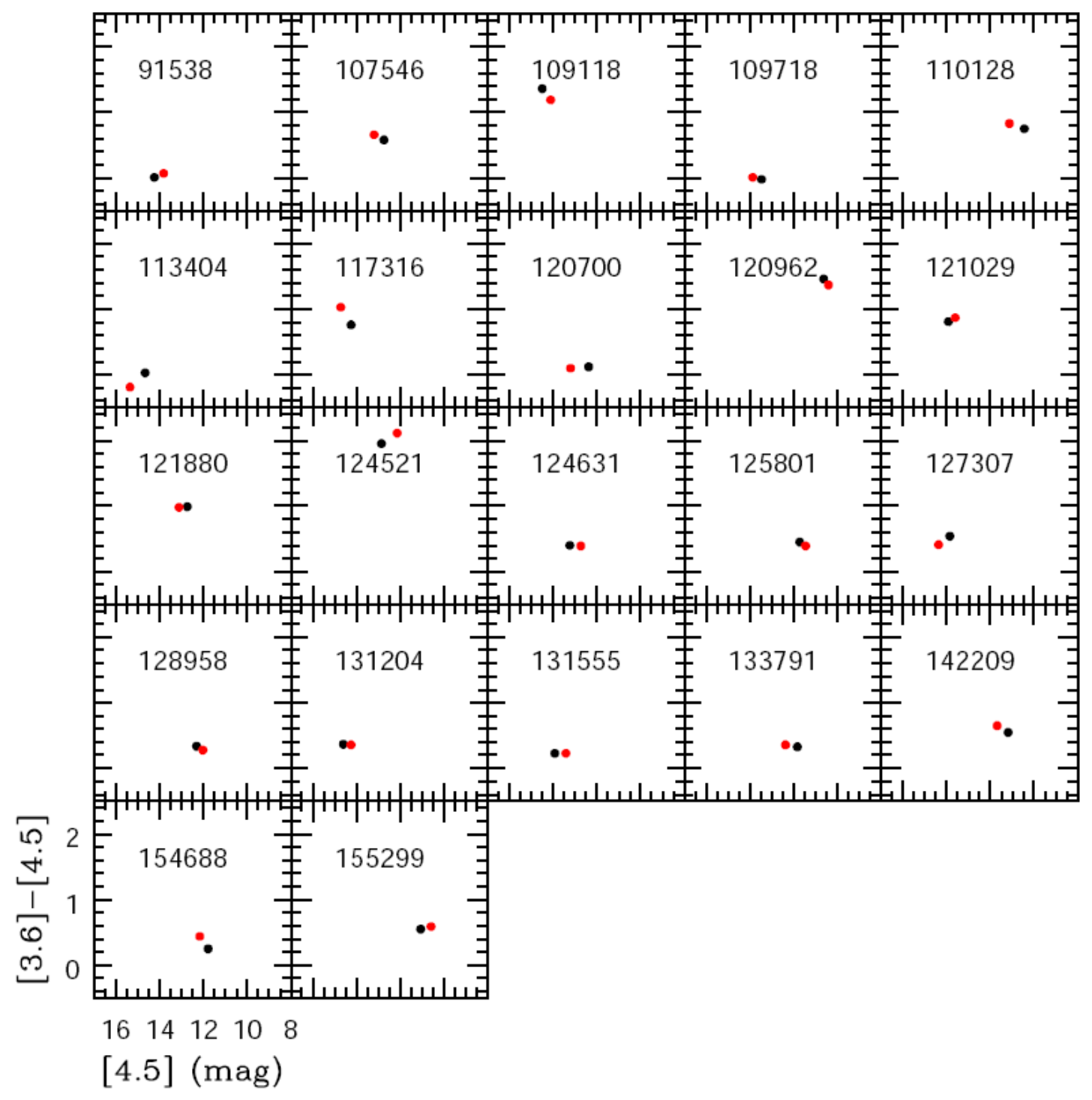

Fig. 8.- b). As Figure 8a, continued. 


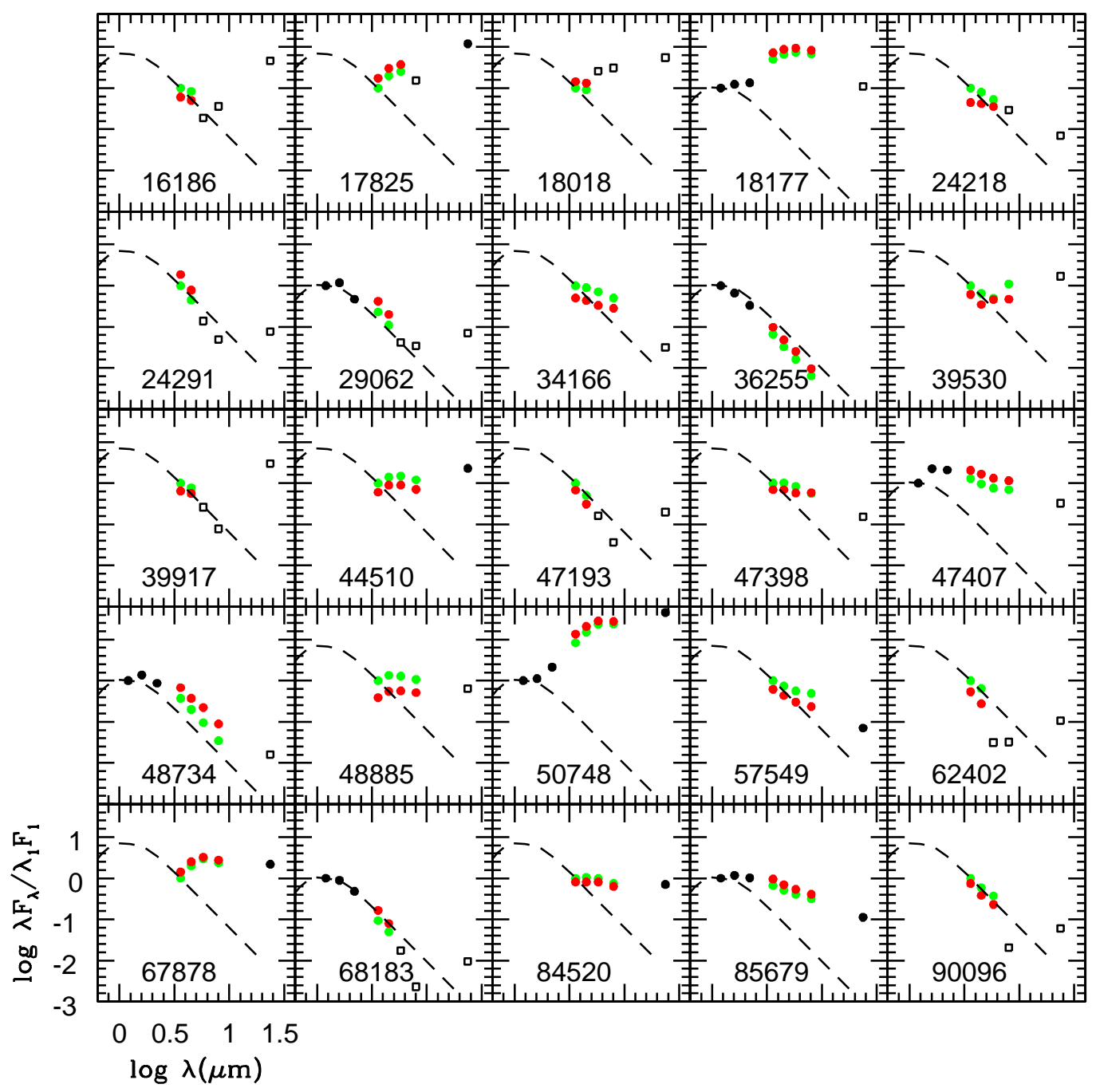

Fig. 9.- a). SED's of sources as numbered in Table 2. IRAC fluxes observed in the first (second) epoch are depicted as green (red) dots. When available, 2MASS fluxes are also plotted for completeness although obtained about seven years prior to the Spitzer advent. MIPS fluxes (at $24 \mu \mathrm{m}$ ) are also not strictly simultaneous to IRAC ones. 2MASS and MIPS $24 \mu \mathrm{m}$ fluxes are plotted as black dots, while open squares indicate $3 \sigma$ upper limits. Dashed line represents a median photosphere of stars in the spectral range K5-M5. As indicated by the ordinate label, all the data are normalized to the flux corresponding to the shortest available wavelength, namely $\mathrm{J}$ or $3.6 \mu \mathrm{m}$ (this latter for the sources without a 2MASS counterpart). 


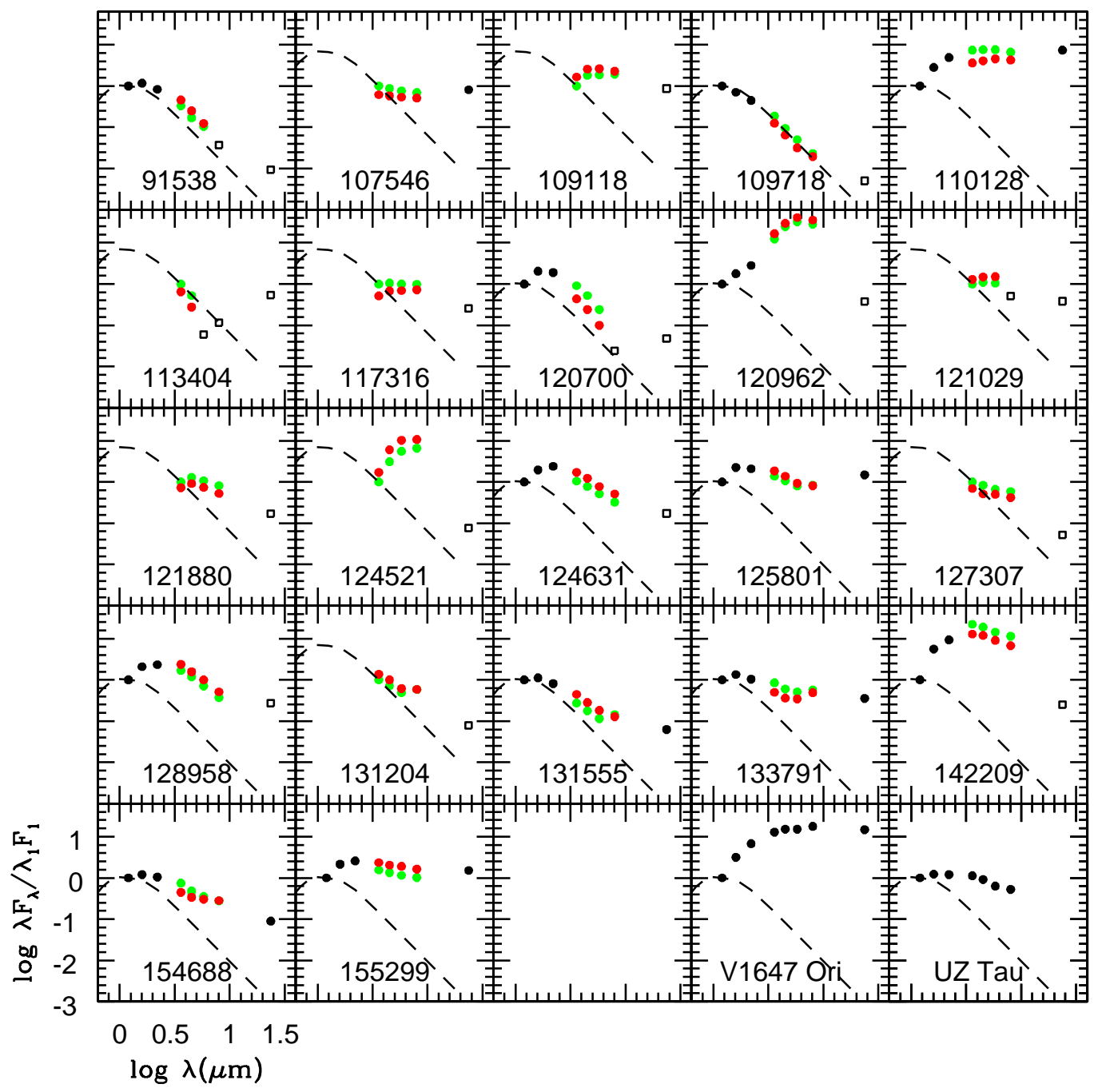

Fig. 9.- b). As in Figure 9a. SED's of V1647 Ori and UZ Tau are shown in the last two panels for comparative purposes. 
Table 1. Statistics of IRAC variables.

\begin{tabular}{cccccc}
\hline \hline Band & $\mathrm{N}_{e p}{ }^{a}$ & $\mathrm{~N}_{m}{ }^{b}$ & $\mathrm{~N}_{\text {var }}{ }^{c}$ & $\mathrm{~N}_{\text {dec }}{ }^{d}$ & $\mathrm{~N}_{\text {ris }}{ }^{e}$ \\
\hline $3.6 \mu \mathrm{m}$ & 138601 & 40382 & 306 & 190 & 116 \\
$4.5 \mu \mathrm{m}$ & 126901 & 27519 & 201 & 108 & 93 \\
$5.8 \mu \mathrm{m}$ & 24256 & 6751 & 121 & 65 & 56 \\
$8.0 \mu \mathrm{m}$ & 12977 & 3207 & 84 & 45 & 39 \\
3.6 and $4.5 \mu \mathrm{m}$ & 114515 & 24482 & 53 & - & - \\
\hline \hline
\end{tabular}

a - Number of sources detected in the first epoch (this number does not change significantly in the second epoch).

b - Number of sources whose magnitude is less than a given threshold (16, 15.5, 13.5 and 12.5 mag in band $1,2,3$, and 4 , respectively).

c - Number of sources selected as variables (see text)

d - Number of declining sources $\left(\operatorname{mag}_{e p 1}-\operatorname{mag}_{e p 2}<0\right)$

e - Number of rising sources $\left(\operatorname{mag}_{e p 1}-\operatorname{mag}_{e p 2}>0\right)$ 
Table 2. Magnitudes of the selected variables.

\begin{tabular}{|c|c|c|c|c|c|c|c|c|c|c|}
\hline ident. & $\alpha(2000)$ & $\delta(2000)$ & $\mathrm{J}$ & $\mathrm{H}$ & K & {$[3.6]$} & {$[4.5]$} & {$[5.8]$} & {$[8.0]$} & {$[24]$} \\
\hline \multirow[t]{2}{*}{16186} & 84529.7 & -435048.1 & - & - & - & 13.50 & 12.96 & - & - & - \\
\hline & & & - & - & - & 14.04 & 13.51 & - & - & - \\
\hline \multirow[t]{2}{*}{17825} & 84533.9 & -43523.0 & - & - & - & 11.91 & 10.44 & 9.43 & - & - \\
\hline & & & - & - & - & 11.32 & 9.99 & 9.01 & - & - \\
\hline \multirow[t]{2}{*}{18018} & 84534.4 & -43503.8 & - & - & - & 13.12 & 12.49 & - & - & - \\
\hline & & & - & - & - & 12.74 & 12.10 & - & - & - \\
\hline \multirow[t]{2}{*}{18177} & 84534.8 & -435257.6 & 16.69 & 15.71 & 14.77 & 11.93 & 10.91 & 10.02 & 9.14 & - \\
\hline & & & - & - & - & 11.53 & 10.60 & 9.76 & 8.91 & - \\
\hline \multirow[t]{2}{*}{24218} & 84549.8 & -435656.5 & - & - & - & 14.96 & 14.46 & 14.15 & - & - \\
\hline & & & - & - & - & 15.82 & 15.16 & 14.58 & - & - \\
\hline \multirow[t]{2}{*}{24291} & 84550.0 & -435650.6 & - & - & - & 15.03 & 15.16 & - & - & - \\
\hline & & & - & - & - & 14.36 & 14.58 & - & - & - \\
\hline \multirow[t]{2}{*}{29062} & 84601.5 & $\begin{array}{lll}-43 & 41 & 20.2\end{array}$ & 16.37 & 15.43 & 15.60 & 14.95 & 15.02 & - & - & - \\
\hline & & & - & - & - & 14.55 & 14.62 & - & - & - \\
\hline \multirow[t]{2}{*}{34166} & 84614.1 & -432348.0 & - & - & - & 13.11 & 12.52 & 12.02 & 11.39 & - \\
\hline & & & - & - & - & 13.87 & 13.31 & 12.83 & 12.04 & - \\
\hline \multirow[t]{2}{*}{36255} & 84619.2 & -43519.4 & 11.52 & 11.22 & 11.13 & 11.46 & 11.49 & 11.48 & 11.49 & - \\
\hline & & & - & - & & 11.02 & 11.06 & 11.02 & 11.09 & - \\
\hline \multirow[t]{2}{*}{39530} & 84627.1 & -435431.2 & - & - & - & 14.68 & 14.43 & 13.97 & 12.10 & - \\
\hline & & & - & - & - & 15.20 & 15.09 & 14.05 & 13.03 & - \\
\hline \multirow[t]{2}{*}{39917} & 84628.1 & -435431.9 & - & - & - & 14.96 & 14.52 & - & - & - \\
\hline & & & - & - & - & 15.46 & 14.86 & - & - & - \\
\hline \multirow[t]{2}{*}{44510} & 84639.5 & -432250.5 & - & - & - & 13.24 & 12.16 & 11.33 & 10.58 & 6.31 \\
\hline & & & - & - & - & 13.80 & 12.64 & 11.89 & 11.14 & - \\
\hline \multirow[t]{2}{*}{47193} & 84646.2 & -435024.5 & - & - & - & 14.46 & 14.47 & - & - & - \\
\hline & & & - & - & - & 14.86 & 14.49 & - & - & - \\
\hline \multirow[t]{2}{*}{47398} & 84646.7 & -431959.3 & - & - & - & 14.57 & 13.81 & 13.28 & 12.74 & - \\
\hline & & & - & - & - & 14.97 & 14.24 & 13.68 & 12.67 & - \\
\hline \multirow[t]{2}{*}{47407} & 84646.7 & -435252.4 & 16.68 & 15.08 & 14.30 & 13.41 & 13.00 & 12.49 & 11.60 & - \\
\hline & & & - & - & - & 12.91 & 12.39 & 11.89 & 11.06 & - \\
\hline \multirow[t]{2}{*}{48734} & 84649.9 & -43191.2 & 15.69 & 14.59 & 14.26 & 13.75 & 13.71 & 13.74 & 13.86 & - \\
\hline & & & - & - & - & 13.10 & 13.03 & 12.82 & 12.83 & - \\
\hline \multirow[t]{2}{*}{48885} & 84650.3 & -435257.0 & - & - & - & 14.73 & 13.68 & 12.96 & 12.17 & - \\
\hline & & & - & - & - & 15.74 & 14.65 & 13.87 & 12.98 & - \\
\hline \multirow[t]{2}{*}{50748} & 84654.7 & -435236.3 & 17.37 & 16.50 & 14.97 & 12.04 & 10.68 & 9.45 & 8.42 & 4.16 \\
\hline & & & - & - & - & 11.52 & 10.32 & 9.24 & 8.27 & - \\
\hline \multirow[t]{2}{*}{57549} & 8479.8 & -435631.7 & - & - & - & 13.06 & 12.64 & 12.17 & 11.36 & 9.88 \\
\hline & & & - & - & - & 13.58 & 13.22 & 12.87 & 12.16 & - \\
\hline \multirow[t]{2}{*}{62402} & 84719.8 & $\begin{array}{lll}-43 & 21 & 20.4\end{array}$ & - & - & - & 14.79 & 14.54 & - & - & - \\
\hline & & & - & - & - & 15.47 & 15.46 & - & - & - \\
\hline 67878 & 84730.6 & -432822.4 & - & - & - & 13.14 & 11.68 & 10.48 & 9.74 & 6.25 \\
\hline & & & - & - & - & 12.77 & 11.43 & 10.38 & 9.58 & - \\
\hline 68183 & 84731.3 & -43553.1 & 15.37 & 14.76 & 14.61 & 14.96 & 14.90 & - & - & - \\
\hline & & & - & - & - & 14.33 & 14.40 & - & - & - \\
\hline 84520 & 8480.4 & -432037.2 & - & - & - & 13.36 & 12.59 & 11.91 & 11.22 & 7.70 \\
\hline & & & - & - & - & 13.61 & 12.86 & 12.12 & 11.40 & - \\
\hline
\end{tabular}


Table 2-Continued

\begin{tabular}{|c|c|c|c|c|c|c|c|c|c|c|}
\hline ident. & $\alpha(2000)$ & $\delta(2000)$ & $\mathrm{J}$ & $\mathrm{H}$ & $\mathrm{K}$ & {$[3.6]$} & {$[4.5]$} & {$[5.8]$} & {$[8.0]$} & {$[24]$} \\
\hline \multirow[t]{2}{*}{85679} & 8482.3 & -431850.5 & 14.34 & 13.42 & 12.73 & 11.77 & 11.33 & 10.80 & 10.09 & 7.66 \\
\hline & & & - & - & - & 11.37 & 10.99 & 10.50 & 9.84 & - \\
\hline \multirow[t]{2}{*}{90096} & 84810.0 & -431957.9 & - & - & - & 14.39 & 14.23 & 13.96 & - & - \\
\hline & & & - & - & - & 14.70 & 14.70 & 14.48 & - & - \\
\hline \multirow[t]{2}{*}{91538} & 84812.6 & -435917.4 & 16.05 & 15.15 & 14.69 & 14.26 & 14.25 & 14.01 & - & - \\
\hline & & & - & - & - & 13.90 & 13.83 & 13.83 & - & - \\
\hline \multirow[t]{2}{*}{107546} & 84842.8 & -431732.1 & - & - & - & 13.32 & 12.74 & 12.13 & 11.25 & 7.52 \\
\hline & & & - & - & - & 13.86 & 13.20 & 12.50 & 11.57 & - \\
\hline \multirow[t]{2}{*}{109118} & 84846.0 & -433727.0 & - & - & - & 15.87 & 14.51 & 13.71 & 12.69 & - \\
\hline & & & - & - & - & 15.31 & 14.12 & 13.33 & 12.50 & - \\
\hline \multirow[t]{2}{*}{109718} & 84847.3 & -434557.4 & 14.64 & 14.26 & 13.94 & 13.45 & 13.47 & 13.39 & 13.26 & - \\
\hline & & & - & - & - & 13.88 & 13.87 & 13.89 & 13.43 & - \\
\hline \multirow[t]{2}{*}{110128} & 84848.1 & -435618.1 & 16.39 & 14.52 & 13.08 & 11.20 & 10.45 & 9.69 & 8.86 & 5.15 \\
\hline & & & - & - & - & 11.96 & 11.13 & 10.23 & 9.32 & - \\
\hline \multirow[t]{2}{*}{113404} & 8480.4 & -432037.2 & - & - & - & 14.70 & 14.67 & - & - & - \\
\hline & & & - & - & - & 15.17 & 15.36 & - & - & - \\
\hline \multirow[t]{2}{*}{117316} & 8494.1 & -43202.4 & - & - & - & 15.01 & 14.25 & 13.55 & 12.58 & - \\
\hline & & & - & - & - & 15.75 & 14.72 & 13.94 & 12.91 & - \\
\hline \multirow[t]{2}{*}{120700} & 84912.4 & $-4317 \quad 14.4$ & 15.41 & 13.89 & 13.13 & 12.50 & 12.38 & 12.46 & - & - \\
\hline & & & - & - & - & 13.31 & 13.21 & 13.40 & - & - \\
\hline \multirow[t]{2}{*}{120962} & 84913.0 & -433548.1 & 17.83 & 16.47 & 15.12 & 12.09 & 10.63 & 9.56 & 8.73 & - \\
\hline & & & - & - & - & 11.78 & 10.41 & 9.32 & 8.49 & - \\
\hline \multirow[t]{2}{*}{121029} & 84913.2 & -433638.4 & - & - & - & 14.74 & 13.93 & 13.21 & - & - \\
\hline & & & - & - & - & 14.48 & 13.61 & 12.82 & - & - \\
\hline \multirow[t]{2}{*}{121880} & 84915.2 & -433633.2 & - & - & - & 13.74 & 12.75 & 12.18 & 11.51 & - \\
\hline & & & - & - & - & 14.10 & 13.12 & 12.57 & 11.97 & - \\
\hline \multirow[t]{2}{*}{124521} & 84921.8 & -4421.3 & - & - & - & 14.81 & 12.86 & 11.47 & 10.31 & - \\
\hline & & & - & - & - & 14.25 & 12.14 & 10.81 & 9.78 & - \\
\hline \multirow[t]{2}{*}{124631} & 84922.1 & -433542.5 & 16.70 & 15.24 & 14.19 & 13.64 & 13.24 & 12.94 & 12.46 & - \\
\hline & & & - & - & - & 13.13 & 12.74 & 12.49 & 11.96 & - \\
\hline \multirow[t]{2}{*}{125801} & 84925.0 & -433612.5 & 15.53 & 13.93 & 13.17 & 12.18 & 11.73 & 11.29 & 10.26 & 6.06 \\
\hline & & & - & - & - & 11.84 & 11.45 & 11.11 & 10.27 & - \\
\hline \multirow[t]{2}{*}{127307} & 84928.7 & -433829.1 & - & - & - & 14.39 & 13.85 & 13.32 & 12.49 & - \\
\hline & & & - & - & - & 14.78 & 14.37 & 13.63 & 12.85 & - \\
\hline \multirow[t]{2}{*}{128958} & 84932.7 & $-43 \quad 1514.2$ & 16.25 & 14.71 & 13.76 & 12.66 & 12.32 & 12.13 & 11.86 & - \\
\hline & & & - & - & - & 12.31 & 12.03 & 11.75 & 11.51 & - \\
\hline \multirow[t]{2}{*}{131204} & 84938.2 & -435143.0 & - & - & - & 14.97 & 14.60 & 14.27 & 13.09 & - \\
\hline & & & - & - & - & 14.61 & 14.25 & 14.00 & 13.08 & - \\
\hline \multirow[t]{2}{*}{131555} & 84939.1 & -43395.3 & 15.78 & 14.91 & 14.43 & 14.16 & 13.93 & 13.64 & 12.41 & 9.74 \\
\hline & & & - & - & - & 13.65 & 13.42 & 13.15 & 12.53 & - \\
\hline 133791 & 84944.5 & -433448.8 & 15.01 & 13.93 & 13.38 & 12.16 & 11.83 & 11.23 & 10.14 & 7.09 \\
\hline & & & - & - & - & 12.74 & 12.38 & 11.66 & 10.30 & - \\
\hline 142209 & 8504.6 & -432351.3 & 18.13 & 15.52 & 14.13 & 11.74 & 11.19 & 10.73 & 10.01 & - \\
\hline & & & - & - & - & 12.34 & 11.69 & 11.23 & 10.59 & - \\
\hline 154688 & 85034.1 & $-4352 \quad 1.1$ & 14.74 & 13.78 & 13.12 & 12.04 & 11.79 & 11.36 & 10.65 & 8.32 \\
\hline & & & - & - & - & 12.61 & 12.17 & 11.55 & 10.62 & - \\
\hline
\end{tabular}


Table 2-Continued

\begin{tabular}{ccccccccccc}
\hline \hline ident. & $\alpha(2000)$ & $\delta(2000)$ & $\mathrm{J}$ & $\mathrm{H}$ & $\mathrm{K}$ & {$[3.6]$} & {$[4.5]$} & {$[5.8]$} & {$[8.0]$} & {$[24]$} \\
\hline \multirow{2}{*}{155299} & \multirow{2}{*}{5035.5} & -433335.9 & 15.12 & 13.55 & 12.51 & 11.61 & 11.06 & 10.46 & 9.60 & 5.61 \\
& & & - & - & - & 11.18 & 10.59 & 9.92 & 9.10 & - \\
\hline \hline
\end{tabular}

- Source identification refers to our internal numbering (Strafella et al. 2009).

- All the values are given in magnitudes. Conversion into flux densities (Jansky) can be done by means of the zero-mag fluxes that are: $280.9 \mathrm{Jy}(3.6 \mu \mathrm{m}), 179.7 \mathrm{Jy}(4.5 \mu \mathrm{m}), 115.0 \mathrm{Jy}(5.8 \mu \mathrm{m}), 64.13 \mathrm{Jy}$ $(8.0 \mu \mathrm{m})$, and $7.17 \mathrm{Jy}(24 \mu \mathrm{m})$.

- Typical errors (in mag) are: 2MASS, J: 0.02-0.18, H: 0.02-0.17, K: 0.02-0.15; IRAC, all bands: 0.1; MIPS $24 \mu \mathrm{m}: 0.3$.

- Magnitudes in other bands:

- 36255: $\mathrm{B}=14.0, \mathrm{~V}=13.2, \mathrm{R}=12.8$

- 47193: $\mathrm{B}=18.8, \mathrm{R}=17.6$

- 68183: $\mathrm{B}=13.3, \mathrm{~V}=17.7, \mathrm{R}=17.6$

- 85675: $\mathrm{B}=19.5, \mathrm{R}=18.2$

- 91538: R:19.9, I=18.2

- 109718: $\mathrm{B}=18.3, \mathrm{~V}=16.7, \mathrm{R}=16.1$

- 121029: MIPS70=-1.7

- 124521: MIPS70 $=0.43$

- 131555: $\mathrm{B}=21.2, \mathrm{R}=20.0$

- 133791: $\mathrm{B}=20.9, \mathrm{R}=19.0$

- 154688: $\mathrm{B}=19.5, \mathrm{R}=17.7$

- 155299: R(phot $\mathrm{F})=19.5$ 
Table 3. Coded photometric properties of the selected sources.

\begin{tabular}{|c|c|c|c|c|c|c|}
\hline Id. ${ }^{a}$ & location $^{b}$ & $\begin{array}{c}\text { YSO vs. } \\
\text { other }\end{array}$ & $\begin{array}{l}\Delta \operatorname{mag}^{c} \\
\text { vs. } \lambda\end{array}$ & $\begin{array}{c}{[3.6]-[4.5]^{d}} \\
\quad \text { vs. }[4.5]\end{array}$ & $\mathcal{E}^{e}$ & $\begin{array}{c}\mathrm{L}_{I R A C} \\
\left(\mathrm{~L}_{\odot}\right)\end{array}$ \\
\hline 16186 & $\operatorname{IRS} 16^{f}$ & YSO & $\mathrm{C}$ & 0 & 0.7 & 0.004 \\
\hline 17825 & $\operatorname{IRS} 16^{f}$ & YSO & $\mathrm{D}$ & - & 4.1 & 0.101 \\
\hline 18018 & IRS16 $6^{f}$ & YSO & $\mathrm{C}$ & 0 & 1.2 & 0.008 \\
\hline 18177 & $\operatorname{IRS} 16^{f}$ & YSO & $\mathrm{D}$ & - & 20.0 & 0.072 \\
\hline 24218 & $\mathrm{I}$ & AGB/XGAL & $\mathrm{D}$ & - & 1.0 & 0.001 \\
\hline 24291 & I & PHT & $\mathrm{D}$ & - & 1.0 & 0.001 \\
\hline 29062 & $\mathrm{O}$ & XGAL & $\mathrm{C}$ & 0 & 1.7 & 0.001 \\
\hline 34166 & $\mathrm{O}$ & YSO & $\mathrm{C}$ & 0 & 1.5 & 0.009 \\
\hline 36255 & I & PHT/AGB & $\mathrm{C}$ & 0 & 1.0 & 0.037 \\
\hline 39530 & $\mathrm{P}$ & XGAL & I & + & 2.0 & 0.002 \\
\hline 39917 & $\mathrm{P}$ & PHT & $\mathrm{D}$ & - & 1.0 & 0.001 \\
\hline 44510 & I & YSO & $\mathrm{C}$ & - & 3.0 & 0.015 \\
\hline 47193 & I & PHT & I & + & 1.0 & 0.001 \\
\hline 47398 & I & YSO & $\mathrm{C}$ & 0 & 1.5 & 0.003 \\
\hline 47407 & $\mathrm{P}$ & YSO & $\mathrm{C}$ & + & 4.6 & 0.011 \\
\hline 48734 & I & PHT & I & 0 & 2.4 & 0.006 \\
\hline 48885 & $\mathrm{P}$ & YSO & $\mathrm{D}$ & - & 3.0 & 0.003 \\
\hline 50748 & $\mathrm{P}$ & YSO & $\mathrm{D}$ & - & 56.0 & 0.107 \\
\hline 57549 & $\mathrm{O}$ & AGB & I & + & 1.5 & 0.009 \\
\hline 62402 & I & PHT & I & + & 1.0 & 0.001 \\
\hline 67878 & $\mathrm{P}$ & YSO & $\mathrm{D}$ & - & 6.3 & 0.038 \\
\hline 68183 & I & PHT & $\mathrm{D}$ & - & 1.2 & 0.001 \\
\hline 84520 & I & YSO & $\mathrm{C}$ & 0 & 2.2 & 0.011 \\
\hline 85679 & I & AGB & $\mathrm{D}$ & - & 2.6 & 0.045 \\
\hline 90096 & I & PHT & I & + & 1.0 & 0.002 \\
\hline 91538 & $\mathrm{O}$ & PHT/AGB & $\mathrm{D}$ & + & 1.7 & 0.003 \\
\hline 107546 & $\mathrm{P}$ & YSO & $\mathrm{D}$ & - & 1.8 & 0.009 \\
\hline 109118 & I & YSO & $\mathrm{D}$ & - & 6.6 & 0.003 \\
\hline 109718 & I & PHT/AGB & $\mathrm{D}$ & 0 & 1.2 & 0.004 \\
\hline 110128 & I & YSO & $\mathrm{D}$ & - & 16.0 & 0.072 \\
\hline 113404 & I & XGAL & I & + & 1.0 & 0.001 \\
\hline 117316 & $\mathrm{P}$ & XGAL & $\mathrm{D}$ & - & 2.0 & 0.002 \\
\hline 120700 & $\mathrm{P}$ & PHT & I & 0 & 2.9 & 0.009 \\
\hline 120962 & $\mathrm{P}$ & YSO & $\mathrm{C}$ & - & 50.0 & 0.096 \\
\hline 121029 & $\mathrm{P}$ & YSO & I & + & 3.0 & 0.004 \\
\hline 121880 & $\mathrm{P}$ & YSO & $\mathrm{C}$ & 0 & 2.7 & 0.008 \\
\hline
\end{tabular}


Table 3-Continued

\begin{tabular}{lcccccc}
\hline \hline \multicolumn{1}{c}{ Id. $^{a}$} & location $^{b}$ & $\begin{array}{c}\text { YSO vs. } \\
\text { other }\end{array}$ & $\begin{array}{c}\Delta \text { mag }^{c} \\
\text { vs. } \lambda\end{array}$ & $\begin{array}{c}{[3.6]-[4.5]^{d}} \\
\text { vs. }[4.5]\end{array}$ & $\mathcal{E}^{e}$ & $\begin{array}{c}\mathrm{L}_{I R A C} \\
\left(\mathrm{~L}_{\odot}\right)\end{array}$ \\
\hline 124521 & $\mathrm{I}$ & YSO & $\mathrm{C}$ & + & 20.0 & 0.020 \\
$\mathbf{1 2 4 6 3 1}$ & $\mathrm{P}$ & YSO & $\mathrm{C}$ & 0 & 5.0 & 0.008 \\
$\mathbf{1 2 5 8 0 1}$ & $\mathrm{I}$ & YSO & $\mathrm{D}$ & - & 4.6 & 0.029 \\
127307 & $\mathrm{O}$ & YSO & $\mathrm{C}$ & + & 1.5 & 0.003 \\
128958 & $\mathrm{I}$ & PHT & $\mathrm{C}$ & - & 4.8 & 0.016 \\
131204 & $\mathrm{O}$ & XGAL & $\mathrm{D}$ & 0 & 2.0 & 0.002 \\
$\mathbf{1 3 1 5 5 5}$ & $\mathrm{I}$ & YSO & $\mathrm{D}$ & 0 & 2.0 & 0.004 \\
$\mathbf{1 3 3 7 9 1}$ & $\mathrm{I}$ & YSO & $\mathrm{D}$ & 0 & 2.7 & 0.022 \\
$\mathbf{1 4 2 2 0 9}$ & $\mathrm{P}$ & YSO & $\mathrm{C}$ & - & 42.0 & 0.033 \\
154688 & $\mathrm{O}$ & AGB & $\mathrm{D}$ & - & 2.4 & 0.022 \\
155299 & $\mathrm{O}$ & YSO & $\mathrm{C}$ & 0 & 5.9 & 0.064 \\
\hline EXor class & $\mathrm{P}(\mathrm{I})$ & YSO & $\mathrm{D}(\mathrm{C})$ & $-(0)$ & $>1.0$ & \\
\hline
\end{tabular}

a In bold-face the candidate young variables are indicated

b $\mathrm{O}=$ outside the $\mathrm{CO}$ contours, $\mathrm{I}=$ inside, $\mathrm{P}=$ on a peak of warm gas.

c $\quad \mathrm{D}=$ decreasing, $\mathrm{C}=$ constant, $\mathrm{I}=$ increasing.

d $-=$ redder, $0=$ constant,$+=$ bluer color during a fading event.

e ratio of the observed SED and the underlying normalized K5-M5 photosphere, both integrated from $J$ to $8 \mu \mathrm{m}$.

f denotes that the source lies within the IR cluster IRS16, although located outside our CO map. 